\title{
Theoretical study on finite-thickness effect on harmonics in Richtmyer-Meshkov instability for arbitrary Atwood numbers
}

Cite as: Phys. Plasmas 25, 122103 (2018); https://doi.org/10.1063/1.5053766

Submitted: 25 August 2018. Accepted: 08 November 2018. Published Online: 07 December 2018

Wanhai Liu, Xinliang Li, Changping Yu, Yaowei Fu, Pei Wang, Lili Wang, and Wenhua Ye
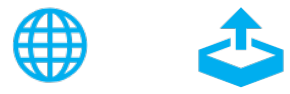

\section{ARTICLES YOU MAY BE INTERESTED IN}

Progress toward a self-consistent set of 1D ignition capsule metrics in ICF

Physics of Plasmas 25, 122704 (2018); https://doi.org/10.1063/1.5049595

Optimization of laser-driven cylindrical implosions on the OMEGA laser Physics of Plasmas 25, 122701 (2018); https://doi.org/10.1063/1.5055776

Wavelet methods for studying the onset of strong plasma turbulence

Physics of Plasmas 25, 122310 (2018); https://doi.org/10.1063/1.5062853

\section{Where in the world is AIP Publishing?}

Find out where we are exhibiting next 


\title{
Theoretical study on finite-thickness effect on harmonics in Richtmyer-Meshkov instability for arbitrary Atwood numbers
}

\author{
Wanhai Liu, ${ }^{1,2}$ Xinliang Li, ${ }^{2,3}$ Changping Yu, ${ }^{2,3, a)}$ Yaowei Fu, ${ }^{2,3}$ Pei Wang, ${ }^{4,5}$ Lili Wang, $^{4,5}$ \\ and Wenhua $\mathrm{Ye}^{4,5}$ \\ ${ }^{1}$ Research Center of Computational Physics, Mianyang Normal University, Mianyang 621000, China \\ ${ }^{2}$ LHD, Institute of Mechanics, Chinese Academy of Sciences, Beijing 100190, China \\ ${ }^{3}$ School of Engineering Science, University of Chinese Academy of Sciences, Beijing 100049, China \\ ${ }^{4}$ Institute of Applied Physics and Computational Mathematics, Beijing 100088, China \\ ${ }^{5}$ Center for Applied Physics and Technology, Peking University, Center for Applied Physics and Technology, \\ Peking University, China
}

(Received 25 August 2018; accepted 8 November 2018; published online 7 December 2018)

The finite-thickness effect of two superimposed fluids on harmonics in the Richtmyer-Meshkov instability (RMI) for arbitrary Atwood numbers is investigated by using weakly nonlinear analysis up to the third order. When the thickness of the two fluids tends to be infinity, our results can reproduce the classical results where RMI happens at the interface separating two semi-infinity-thickness fluids of different densities. It is found that the thickness has a large influence on the amplitudes of the first three harmonics compared with those in classical RMI. On the one hand, the thickness effect encourages or reduces the amplitudes of the first three harmonics, and on the other hand, it changes the phases of the second and the third harmonics. Published by AIP Publishing.

https://doi.org/10.1063/1.5053766

\section{INTRODUCTION}

The Richtmyer-Meshkov instability (RMI) usually happens at a corrugated interface separating two fluids of different densities for two cases. When an incident shock has collided with a corrugated interface, or when the interface is with nonuniform vorticities, either initially deposited or supplied by external sources, ${ }^{1,2}$ the interface will be unstable and is named as RMI. ${ }^{3,4}$ RMI plays a key role in numerous fields, such as stellar evolution $^{5}$ and transient events in Earth's magnetosphere, ${ }^{6}$ and astrophysical problems ${ }^{7}$ to applications including inertial confinement fusion (ICF). ${ }^{8}$ Therefore, it is essential to understand and estimate the development of the RMI because of its significance in both fundamental research and engineering applications. The classical RMI is considered to happen at the interface separating two fluids with semi-infinity thickness. However, the RMI generally appears when the two fluids are with finite-thickness. ${ }^{9}$

In classical RMI, when an incident shock travels from a light fluid to a heavy fluid, the incident shock will bifurcate: a reflected wave going back to the light fluid and a refracted wave entering the heavy fluid. At this situation, both the reflected and refracted waves are shocks. After these two shocks leave the interface, the fluids near the interface are regarded as being incompressible, and the potential flow theory can be applied to solve this instability problem at the weakly nonlinear stage. The perturbed interface with an initial small amplitude grows from the linear stage to the weakly nonlinear stage, even to strong nonlinear one, i.e., turbulent mixing process. For the linear and weakly nonlinear stages, harmonics play a key role in the evolution of bubbles and spikes which are fingers of the interface. The bubble (spike) refers to the portion of the light (heavy) fluid entering the heavy (light) fluid. For the interface with a single-mode cosine perturbation,

\footnotetext{
${ }^{\text {a) }}$ Author to whom correspondence should be addressed: cpyu@imech.ac.cn
}

the amplitude of the bubble is equal to that of the spike at the linear stage, while the former is less than the latter at the weakly nonlinear stage. This difference between the evolution amplitudes of the bubble and the spike should attribute to the generation of the high harmonics (the second harmonic, the third harmonic, and so on) and the feedback from the high orders (the third order, the fifth order, and so on).

Within the third-order framework, the weakly nonlinear solution for the initial cosine single-mode perturbation is given as

$$
\begin{aligned}
\eta(x, t)= & \overbrace{\eta_{1,1}(t)+\eta_{3,1}(t)}^{\eta_{1}(t)}] \cos (k x)+\eta_{2}(t) \cos (2 k x) \\
& +\eta_{3}(t) \cos (3 k x),
\end{aligned}
$$

with the amplitudes of the first three harmonics being

$$
\begin{gathered}
\eta_{1,1}(t)=t v_{0}+\varepsilon, \\
\eta_{2}(t)=-\frac{1}{2} A k t^{2} v_{0}^{2}, \\
\eta_{3}(t)=\frac{1}{8}\left[\left(4 A^{2}-1\right) t v_{0}-3 \varepsilon\right] k^{2} t^{2} v_{0}^{2}, \\
\eta_{3,1}(t)=-\frac{1}{24}\left[\left(4 A^{2}+1\right) t v_{0}+3 \varepsilon\right] k^{2} t^{2} v_{0}^{2},
\end{gathered}
$$

where $\eta_{1}=\eta_{1,1}+\eta_{3,1}\left(\eta_{2}\right.$ and $\left.\eta_{3}\right)$ is the amplitude of the fundamental mode (the second and the third harmonics), $k=2 \pi /$ $\lambda, \lambda, \varepsilon$, and $v_{0}$ are the wave number, wavelength, amplitude, and velocity of the initial perturbation at the interface, respectively, Atwood number, $A=\left(\rho_{h}-\rho_{l}\right) /\left(\rho_{h}+\rho_{l}\right)$ with $\rho_{h}\left(\rho_{l}\right)$ being the density of the heavy (light) fluid, and $t$ is the time. It should be noted that up to the third order, just the fundamental mode is corrected by the third order, but the second and the third harmonics are not. Here, the third-order 
feedback to the fundamental mode is always negative (i.e., $\left.\eta_{3,1}=-\left[\left(4 A^{2}+1\right) t v_{0}+3 \varepsilon\right] k^{2} t^{2} v_{0}^{2} / 24<0\right)$, reducing the linear growth $\eta_{1,1}=t v_{0}+\varepsilon$ of the fundamental mode, and the amplitude of the second harmonics is also always negative [i.e., $-A k t^{2} v_{0}^{2} / 2<0$ ] for arbitrary Atwood numbers. It is the negative amplitude of the second harmonics that makes the amplitude of the bubble be generally less than that of the spike at the weakly nonlinear stage in planar RMI.

A large number of studies on RMI have been performed, such as some experiments ${ }^{10-12}$ and numerical simulations ${ }^{13-18}$ on the growth rate of the RMI interface and several theories ${ }^{19-28}$ using different explicit methods. Most of these theoretical literature studies are just concerned with the linear growth rate of the interface at the earlier stage and with the asymptotic behavior of the RMI interface fingers: bubbles and spikes.

Differing from the classical RMI, the thickness of the fluids on both sides of the interface is finite. Ref. 29 presented a linear analytic theory of RMI induced by a shock as an impulsive acceleration in an arbitrary number $N$ of stratified fluids. In this paper, we investigate the finite-thickness effect of the fluids on the first three harmonics for the weakly nonlinear stage.

\section{THEORETICAL FRAMEWORK AND EXPLICIT RESULTS UP TO THE THIRD ORDER}

In the Cartesian coordinate system $(x, y, z)$, the heavy fluid with finite-thickness $d_{3}$ is overlapped on the light fluid with finite-thickness $d_{1}$, and there exist three interfaces. Set the middle interface $I_{2}(x, t)$ between them to be at $y=0$ planar, and the upper interface $I_{3}(x, t)$ of the heavy fluid and the lower one $I_{1}(x$, $t$ ) of the light fluid are located at $y=d_{3}$ and $y=-d_{1}$ planar, respectively. For some reasons, these three interfaces are not always planar but with perturbations. To better seek the finitethickness effect, the initial interfaces are given to be

$$
y=I_{n}(x, t=0)=\operatorname{sgn}(n-2) d_{n}+\varepsilon_{n} \cos (k x),
$$

where $n=1,2$, and 3 , the $\operatorname{sgn}(x)$ is the sign function giving -1 , 0 , or 1 depending on whether $x$ is negative, zero, or positive, and $\varepsilon_{1}, \varepsilon_{2}$, and $\varepsilon_{3}$ are perturbation amplitudes of these three interfaces, respectively. Here, the amplitudes of the perturbation are far less than their wavelength, namely, $\max \left(\varepsilon_{1}, \varepsilon_{2}, \varepsilon_{3}\right) \ll \lambda$,

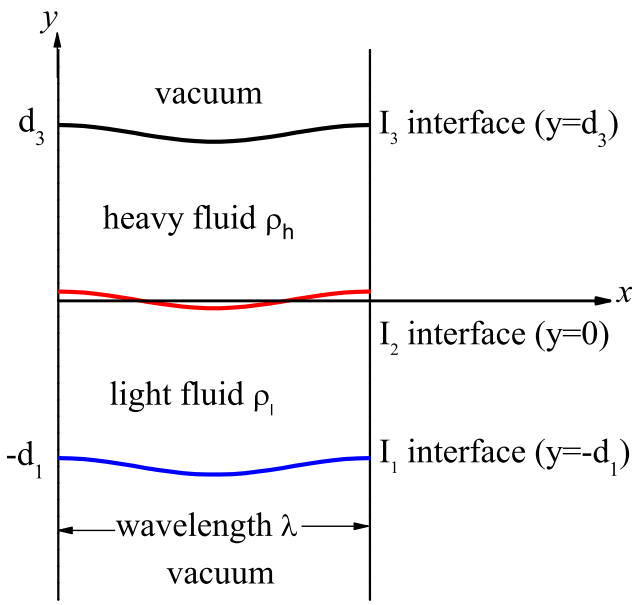

FIG. 1. Schematic figure for the physical situation (of finite thickness fluids between the interfaces). and the regions $y>d_{3}$ and $y<-d_{1}$ are vacuum. The schematic figure for the physical situation is shown in Fig. 1.

After a shock traveling from the light fluid to the heavy one, the initial velocity distribution of these three interfaces is attempted to be

$$
\left.\frac{\partial I_{n}(x, t)}{\partial t}\right|_{t=0}=v_{n} \cos (k x) .
$$

Because of the perturbations of the amplitudes and velocities mentioned above, these three interfaces $I_{1}(x, t)$, $I_{2}(x, t)$, and $I_{3}(x, t)$ evolving with time should satisfy the conditions of the kinematic and pressure boundaries.

For the lower (upper) interface, it is a free boundary: the normal velocities of the interface and light (heavy) fluid should be continuous, while at the middle interface, in the normal direction of this interface, the velocities of the light and heavy fluids should be continuous. Therefore, the conditions of the kinematic boundary are

$$
\frac{\partial I_{n}}{\partial t}+\frac{\partial I_{n}}{\partial x} \frac{\partial \phi_{j}}{\partial x}-\frac{\partial \phi_{j}}{\partial y}=0 \quad \text { at } \quad y=I_{n}(x, t),
$$

where $j=l$ and $j=h$ correspond to $n=1$ and 2 and $n=2$ and 3 , respectively, and the $\phi_{l}$ and $\phi_{h}$ are the velocity potentials of the light fluid and heave fluid, respectively. After a shock travels from the light fluid to the heavy one, the fluids can be viewed as to be incompressible. Hence, $\phi_{l}$ and $\phi_{h}$ should obey the Laplace equation

$$
\frac{\partial^{2} \phi_{j}}{\partial x^{2}}+\frac{\partial^{2} \phi_{j}}{\partial y^{2}}=0 \text { in two fluids }(j=l, h) .
$$

According to the Bernoulli equation, the pressure of the light or heavy fluid without gravitational acceleration is

$$
p_{j}=-\rho_{j}\left(\frac{\partial \phi_{j}}{\partial t}+\frac{1}{2} \nabla \phi_{j} \cdot \nabla \phi_{j}\right)+f_{j}(t),
$$

where $f_{j}(t)$ is an arbitrary function of time. At these three interfaces, the pressure should be continuous. Therefore, the conditions of the equilibrium pressure are written as

$$
\begin{aligned}
& -\rho_{l}\left(\frac{\partial \phi_{l}}{\partial t}+\frac{1}{2} \nabla \phi_{l} \cdot \nabla \phi_{l}\right)+f_{1}(t)=0 \quad \text { at } y=I_{1}(x, t) \\
& -\rho_{h}\left(\frac{\partial \phi_{h}}{\partial t}+\frac{1}{2} \nabla \phi_{h} \cdot \nabla \phi_{h}\right)+f_{3}(t)=0 \quad \text { at } y=I_{3}(x, t), \\
& \rho_{l}\left(\frac{\partial \phi_{l}}{\partial t}+\frac{1}{2} \nabla \phi_{l} \cdot \nabla \phi_{l}\right)-\rho_{h}\left(\frac{\partial \phi_{h}}{\partial t}+\frac{1}{2} \nabla \phi_{h} \cdot \nabla \phi_{h}\right) \\
& \quad+f_{2}(t)=0 \quad \text { at } y=I_{2}(x, t) .
\end{aligned}
$$

Under the framework of the third-order weakly nonlinear theory, the interfaces and velocity potentials at time $t$ normalized by wave number $k$ can be expressed as

$$
\begin{aligned}
\hat{I}_{n}(x, t)= & \sum_{j=1}^{3} \sigma^{j} P_{j, j}(t) \cos (j k x)+\sigma^{3} P_{3,1}(t) \cos (k x) \\
& +\operatorname{sgn}(n-2) \delta_{n},
\end{aligned}
$$




$$
\begin{aligned}
\phi_{l}(x, t)= & \sum_{j=1}^{3} \sigma^{j} \cos (j k x)\left[a_{j, j}(t) e^{j k y}+c_{j, j}(t) e^{-j k y}\right] \\
& +\sigma^{3} \cos (k x)\left[a_{3,1}(t) e^{k y}+c_{3,1}(t) e^{-k y}\right], \\
\phi_{h}(x, t)= & \sum_{j=1}^{3} \sigma^{j} \cos (j k x)\left[b_{j, j}(t) e^{j k y}+q_{j, j}(t) e^{-j k y}\right] \\
& +\sigma^{3} \cos (k x)\left[b_{3,1}(t) e^{k y}+q_{3,1}(t) e^{-k y}\right],
\end{aligned}
$$

where $P$ denotes $L, M$, or $U$ corresponding to $n=1,2$, or 3 , respectively [e.g., when $\mathrm{n}=1$, Eq. (9a) becomes $\hat{I}_{1}(x, t)$ $=\sum_{j=1}^{3} \sigma^{j} L_{j, j}(t) \cos (j k x)+\sigma^{3} L_{3,1}(t) \cos (k x)-\delta_{1}$, and when $\mathrm{n}=2$, Eq. (9a) becomes $\hat{I}_{2}(x, t)=\sum_{j=1}^{3} \sigma^{j} M_{j, j}(t) \cos (j k x)$ $\left.+\sigma^{3} M_{3,1}(t) \cos (k x)\right]$, the normalized parameter $\sigma$ $=\min \left(k \varepsilon_{1}, k \varepsilon_{2}, k \varepsilon_{3}\right) \ll 1$, and the normalized finite thicknesses of the two fluids $\delta_{j}=k d_{j}(j=1,3)$. It should be noted that the velocity potentials $\phi_{l}$ and $\phi_{h}$ have satisfied the Laplace equation (6), and the functions of the interfaces have the relations $\hat{I}_{n}(x, t)=k I_{n}(x, t)(n=1,2$, and 3$)$.

Substitute Eqs. (9a)-(9c) into Eqs. (5) and (8a)-(8c), and then, replace $y$ in these seven resulting equations with the corresponding interface $I_{n}$ expressed above. The final equations containing $x$ and $\sigma$ are obtained.

To further obtain the $m$ th-order $(m>0)$ equations just including the terms of $\sigma^{m}$, we need to expand the left hand sides of these seven final equations in the Maclaurin series of $\sigma$. Here, the zeroth-order equations, considering the effect of arbitrary function $f_{n}(t)$, can be satisfied automatically. Therefore, the first-, second-, and third-order equations together with the initial conditions (3) and (4) can be solved successively. These explicit results are tediously long, especially for the third order. For simplicity, we just give the results for the special case where $\varepsilon_{1}=\varepsilon_{3}=0$ [there are no perturbations at the free boundaries $y=I_{1}(x, t=0)$ and $\left.y=I_{3}(x, t=0)\right]$

$$
\begin{gathered}
M_{1,1}(t)=\frac{1}{\sigma}\left(t v_{0}+\varepsilon_{2}\right), \\
M_{2,2}(t)=-\frac{\left(B_{2}+1\right) A+B_{1}}{2\left(B_{4} A+B_{3}+1\right) \sigma^{2}} k t^{2} v_{0}^{2},
\end{gathered}
$$

$$
\begin{gathered}
M_{3,3}(t)=\frac{\left(C_{0}+1\right)\left[\left(F_{3}+4\right) A^{2}-F_{2} A+F_{1}-1\right] t v_{0}-3\left(F_{6} A^{2}+F_{5} A+F_{4}+1\right) \varepsilon_{2}}{8\left(F_{9} A^{2}+F_{8} A+F_{7}+1\right) \sigma^{3}} k^{2} t^{2} v_{0}^{2}, \\
M_{3,1}(t)=-\frac{\left(C_{0}+1\right)\left[\left(C_{3}+4\right) A^{2}+C_{2} A+C_{1}+1\right] t v_{0}+3\left(C_{6} A^{2}+C_{5} A+C_{4}+1\right) \varepsilon_{2}}{24\left(C_{9} A^{2}+C_{8} A+C_{7}+1\right) \sigma^{3}} k^{2} t^{2} v_{0}^{2},
\end{gathered}
$$

where the finite-thickness factors $B_{1}-B_{4}, C_{0}-C_{9}$, and $F_{1}$ $-F_{9}$ just related to $\delta_{1}$ and $\delta_{3}$, due to their tedious expressions, are shown in the Appendix. It is found that the effect of the finite-thickness fluids does not have an influence on the linear amplitude $\sigma M_{1,1}(t)$ of the fundamental mode, but on the second harmonics $\sigma^{2} M_{2,2}(t)$, the third harmonics $\sigma^{3} M_{3,3}(t)$ and the correction to the fundamental mode $\sigma^{3} M_{3,1}(t)$ from the third order. In a word, the finite-thickness of the fluids is closely related to the first three harmonics, among which the fundamental mode includes its linear amplitude and correctional one. It is obvious that, up to the third order, just the fundamental mode is corrected by the third order, but the second and third harmonics are not. As a result, one can obtain the amplitudes of the first three harmonics with the finite-thickness effect as

$$
\begin{gathered}
\eta_{1}^{t}(t)=\sigma M_{1,1}(t)+\sigma^{3} M_{3,1}(t) \\
\eta_{2}^{t}(t)=\sigma^{2} M_{2,2}(t) \\
\eta_{3}^{t}(t)=\sigma^{3} M_{3,3}(t)
\end{gathered}
$$

and the middle interface is written as

$$
I_{2}(x, t)=\eta_{1}^{t}(t) \cos (k x)+\eta_{2}^{t}(t) \cos (2 k x)+\eta_{3}^{t}(t) \cos (3 k x) .
$$

It should be noted that when the thicknesses $\delta_{1} \rightarrow \infty$ and $\delta_{3}$ $\rightarrow \infty$, the factors $B_{1}-B_{4}, C_{0}-C_{9}$, and $F_{1}-F_{9}$ will tend to be zero, and the middle interface will reproduce the classical evolutional interface (1) separating two semi-infinitethickness fluids.

\section{THE FINITE-THICKNESS EFFECT ON HARMONICS}

For the sake of better investigation on the finite-thickness influence, one needs to normalize the expression of the middle interface by using the parameters $k, \varepsilon_{2}$, and $v_{0}$. Letting both sides of the expression (12) multiplied by $k$ and denoting $X=k x$, $T=k t v_{0}, \hat{\varepsilon}=k \varepsilon_{2}, \hat{\eta}_{j}=k \eta_{j}(j=1,2,3)$, and $\hat{I_{2}}=k I_{2}$, one can obtain the normalized expression of the middle interface

$$
\hat{I}_{2}(X, T)=\hat{\eta}_{1}(T) \cos X+\hat{\eta}_{2}(T) \cos 2 X+\hat{\eta}_{3}(T) \cos 3 X,
$$

where the amplitudes of the first three harmonics are

$$
\hat{\eta}_{1}(T)=(T+\hat{\varepsilon})-\frac{\left(C_{0}+1\right)\left[\left(C_{3}+4\right) A^{2}+C_{2} A+C_{1}+1\right] T+3\left(C_{6} A^{2}+C_{5} A+C_{4}+1\right) \hat{\varepsilon}}{24\left(C_{9} A^{2}+C_{8} A+C_{7}+1\right)} T^{2},
$$




$$
\begin{gathered}
\hat{\eta}_{2}(T)=-\frac{\left(B_{2}+1\right) A+B_{1}}{2\left(B_{4} A+B_{3}+1\right)} T^{2}, \\
\hat{\eta}_{3}(T)=\frac{\left(C_{0}+1\right)\left[\left(F_{3}+4\right) A^{2}-F_{2} A+F_{1}-1\right] T-3\left(F_{6} A^{2}+F_{5} A+F_{4}+1\right) \hat{\varepsilon}}{8\left(F_{9} A^{2}+F_{8} A+F_{7}+1\right)} T^{2} .
\end{gathered}
$$

One finds that the evolutional interface is influenced by the factors of not only Atwood number $A$ and the initial amplitude of the interface perturbations $\hat{\varepsilon}$ but also the finitethicknesses $\delta_{1}$ and $\delta_{3}$. Of course, these factors can also influence the amplitude evolution of the bubbles at $X=2 j \pi$ and spikes at $X=(2 j+1) \pi$ with $j=0,1, \ldots$

In order to illustrate the importance of harmonics in the amplitudes of the bubbles and spikes, we plot cosine functions in Fig. 2 where cosine functions $Y_{1,1}, Y_{2}, Y_{3}$, and $Y_{3,1}$ correspond to the linear harmonics of the fundamental mode, the second, the third, and the correction to the fundamental mode from the third order at some time in this paper, respectively. Up to the third order, the interface is formed of these harmonics [i.e., $Y(X)=Y_{1,1}+Y_{2}+Y_{3}+Y_{3,1}$ ], and the positions of the bubble and the spike are at $X=0$ and $X=\pi$, respectively. When these amplitudes of the harmonics are positive, their contributions to bubbles and spikes are of difference. From Fig. 2, one finds that all the harmonics help the growth of the bubble, while the harmonics $Y_{1,1}, Y_{3}$, and $Y_{3,1}$ at $X=\pi$ are negative, resulting in the increasing spikes, and the harmonic $Y_{2}$ is positive, reducing the growth of the spike. In fact, the fundamental mode and the third harmonics have the same influence on the bubbles and spikes: when their amplitudes are positive, they encourage the bubbles and spikes, and when their amplitudes are negative, they constrain the bubbles and spikes. However, the second harmonics play a key role in the bubbles and spikes. When the amplitude of the second harmonics is positive, it helps bubbles but reduces spikes; when the amplitude is negative, it reduces bubbles and helps spikes. The influences of the amplitude of the first three harmonics on bubbles and spikes are concluded at the following table.

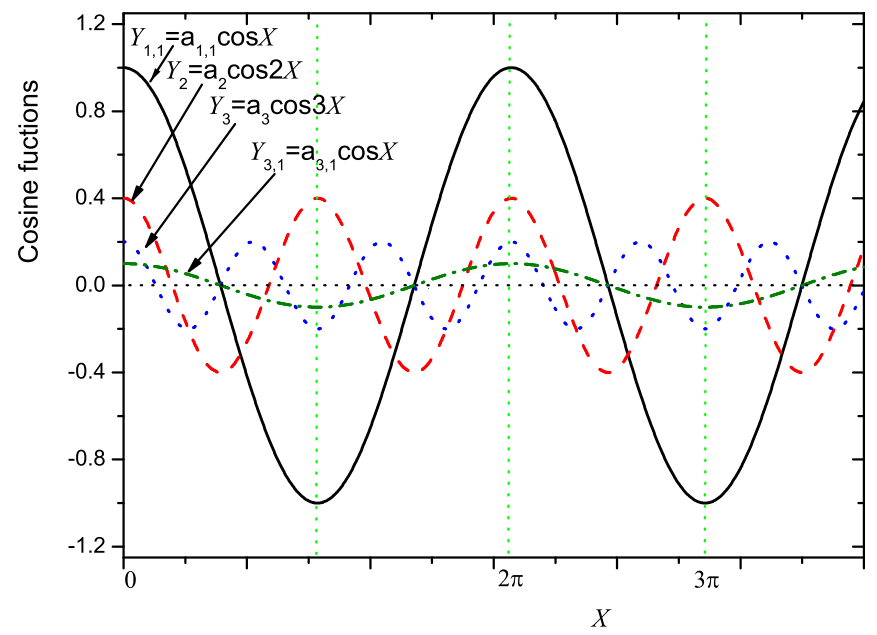

FIG. 2. Cosine functions of $Y_{1,1}=a_{1,1} \cos X, Y_{2}=a_{2} \cos 2 X, Y_{3}=a_{3} \cos 3 X$, and $Y_{3,1}=a_{3,1} \cos X$, where the coefficients $a_{1,1}=1.0, a_{2}=0.4, a_{3}=0.2$, and $a_{3,1}=0.1$.
In Table I, the symbols + and - show the influence of the encouraging and reducing processes on the bubble or the spike, and $A_{b}$ and $A_{s}$ are the amplitudes of the bubble and the spike. It is clear that when the nonlinear effect is not considered (i.e., just keep the linear solution, the fundamental mode), the amplitude of the bubble is equal to that of the spike; while the nonlinearity is in consideration, the amplitude of the bubble is not equal to that of the spike: up to the second order, the former is less than the latter; up to the third order, the amplitudes of the bubble and the spike are strengthened or reduced equally. That is to say, the difference of the amplitudes of the bubble and the spike comes mainly from the second order (i.e., the negative or the positive value of the amplitude of the second harmonic), and the moving speed of the bubble and the spike is closely related to the third order. Therefore, the harmonics play an important role in RMI, especially for the bubble and the spike.

In the following discussion, the normalized initial amplitude of the interface perturbation is fixed as $k \varepsilon=1 / 1000$, and the cases of Atwood numbers $A=0.3$ and $A=0.7$ are selected in this paper. The finite-thickness effect on the evolutions of the first three harmonics is in our consideration for the cases: (1) $\delta_{3} \rightarrow \infty$ and the different finite-thickness $\delta_{1}$; (2) $\delta_{1} \rightarrow \infty$ and the different finite-thickness $\delta_{3}$; and (3) the different $\delta_{1}=\delta_{3}$ which are, respectively, shown in Figs. 3-5.

Figure 3 just considers the finite-thickness effect of the light fluid (i.e., the finite-thicknesses $\delta_{1}$ and $\delta_{3} \rightarrow \infty$ ). It is found that the finite-thickness $\delta_{1}$ has a large influence on amplitudes of the first three harmonics. On the one hand, the finite-thickness influences their amplitudes in size; on the other hand, it can change the phase of the third harmonic. For the first two harmonics, with the decreasing finitethickness $\delta_{1}$, the amplitude of the fundamental mode is constrained, while that of the second harmonics is increased for $A=0.3$ and $A=0.7$. For the larger Atwood number (0.7), when the finite-thickness $\delta_{1}$ decreases, the amplitude of the third harmonics is increased. For $A=0.3$, the amplitude of the third harmonics is positive at $\delta_{1} \leqslant 1$, while it is negative

TABLE I. The influences of the amplitude of the first three harmonics on bubbles and spikes.

\begin{tabular}{lccc}
\hline \hline & Bubble $(X=0)$ & Spike $(X=\pi)$ & $\begin{array}{l}\text { Amplitude relationship } \\
\text { of the bubble and spike }\end{array}$ \\
\hline$a_{1,1}>0$ & + & + & $A_{b}=A_{s}$ \\
$a_{2}>0$ & + & - & $A_{b}>A_{s}$ \\
$a_{2}<0$ & - & + & $A_{b}<A_{s}$ \\
$a_{3}>0$ & + & + & $A_{b}=A_{s}$ \\
$a_{3}<0$ & - & - & $A_{b}=A_{s}$ \\
$a_{3,1}>0$ & + & + & $A_{b}=A_{s}$ \\
$a_{3,1}<0$ & - & - & $A_{b}=A_{s}$ \\
\hline
\end{tabular}


$A=0.3$
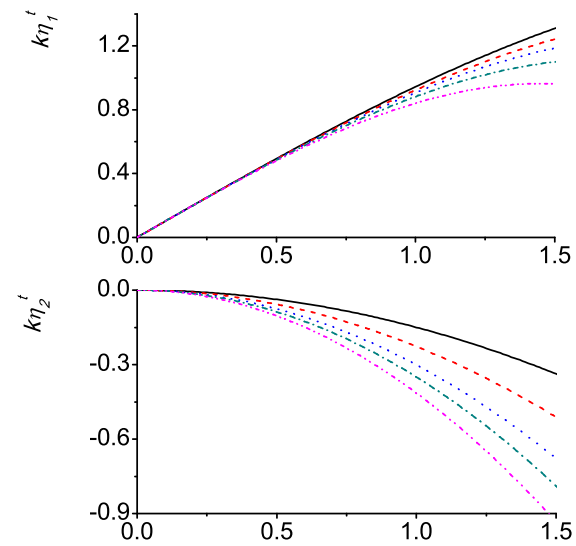

s

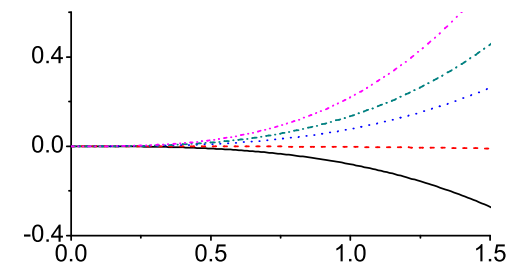

$A=0.7$

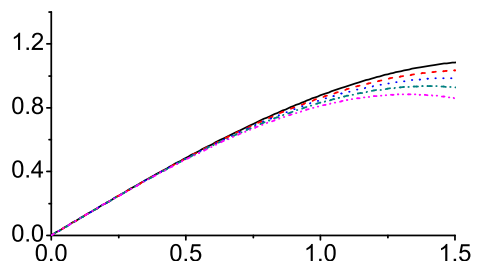

$-\delta_{1}=100$

$\cdots \delta_{1}=2$
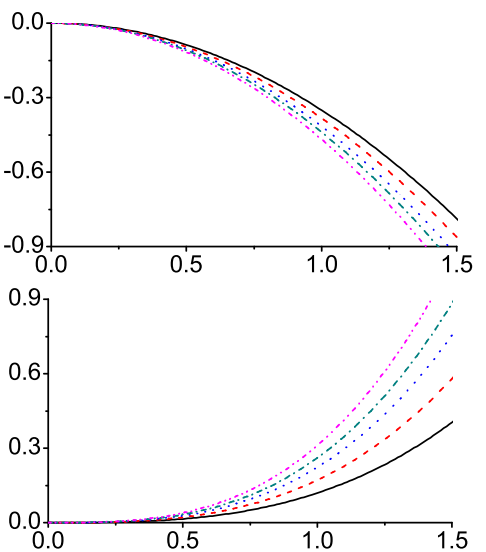

FIG. 3. Normalized amplitudes of the first three harmonics versus normalized time $T=k v_{0} t$ for different finitethicknesses $\delta_{1}=100,2,1,0.5$, and 0.2 of the light fluid and Atwood numbers $A=0.3$ (left) and $A=0.7$ (right). The initial amplitude of the perturbation is $\hat{\varepsilon}=1 / 1000$, and the finite-thickness $\delta_{3} \rightarrow \infty$. at $\delta_{1}>2$. Hence, according to the discussion of the harmonic influence on bubbles and spikes above, the finite-thickness $\delta_{1}$ will result in the amplitude of the spike being far larger than that of the bubble with time, especially for small $\delta_{1}$.

Similar to Fig. 3, Fig. 4 just shows the influence of the finite-thickness $\delta_{3}$ on amplitudes of the first three harmonics. It is found that the finite-thickness $\delta_{3}$ changes the phase of both the second and the third harmonics. The finite-thickness $\delta_{3}$ has a slight influence for small Atwood number $A=0.3$ but increases the amplitude of the fundamental mode for $A=0.7$. For the second harmonic, its amplitude is positive at $\delta_{3} \leqslant 1$ while negative at $\delta_{3} \geqslant 2$. For the third harmonic, its amplitude tends to be zero at $\delta_{3} \leqslant 1$ at $A=0.7$, while it is positive when $\delta_{3} \leqslant 0.5$ and it is negative when $\delta_{3} \geqslant 1$ and $A=0.3$. Therefore, when the finite-thickness $\delta_{3}$ is just taken into account, the amplitude of the spike will not be larger than that of the bubble any longer, depending on the positive amplitude of the second harmonic.

The case of the two fluids with the same finite-thickness $\delta_{1}=\delta_{3}$ is considered in Fig. 5. With the decreasing finitethickness, the amplitudes of the second and the third harmonics for $A=0.3$ and $A=0.7$ will tend to be zero. However, the decreasing thickness mainly influences the amplitude of the fundamental mode especially for $A=0.7$. With the decreasing thickness, the amplitude of the fundamental mode grows linearly with time for $A=0.3$ and $A=0.7$. This shows that the
$A=0.3$
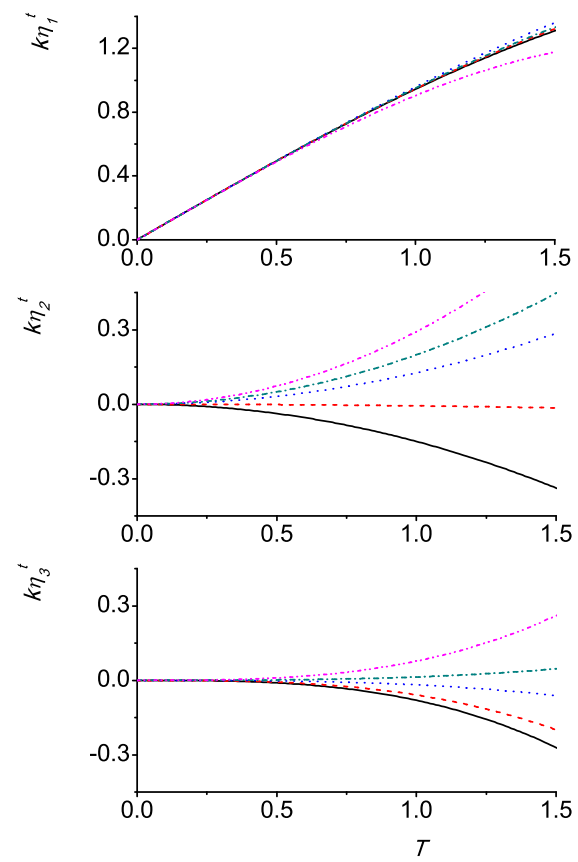

$A=0.7$
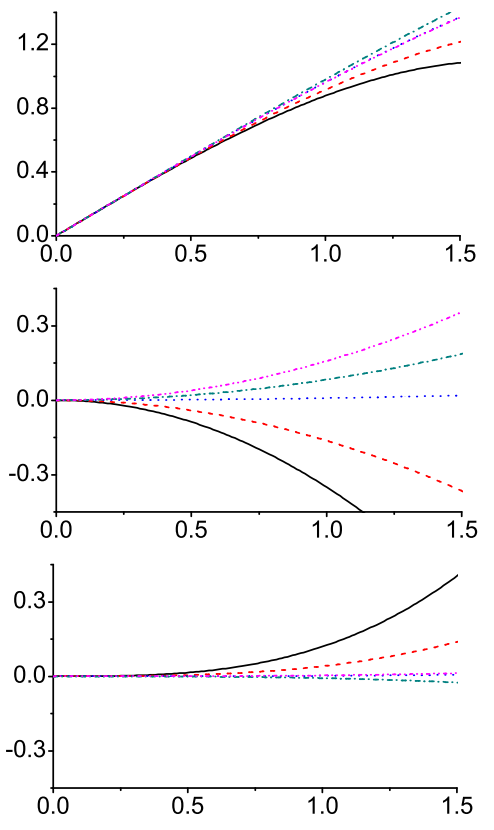

$-\delta_{3}=100$

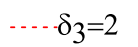

$\cdots \delta_{3}=1$

$-\delta_{3}=0.5$

$\cdots \cdots \delta_{3}=0.2$

$\delta_{1}$ infinity
FIG. 4. Normalized amplitudes of the first three harmonics versus normalized time $T=k v_{0} t$ for different finitethicknesses $\delta_{3}=100,2,1,0.5$, and 0.2 of the heavy fluid and Atwood numbers $A=0.3$ (left) and $A=0.7$ (right). The initial amplitude of the perturbation is $\hat{\varepsilon}=1 / 1000$, and the finitethickness $\delta_{1} \rightarrow \infty$. 
$A=0.3$

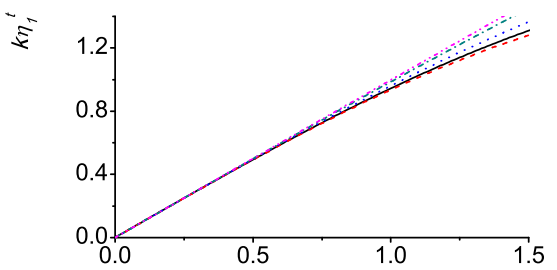

EN

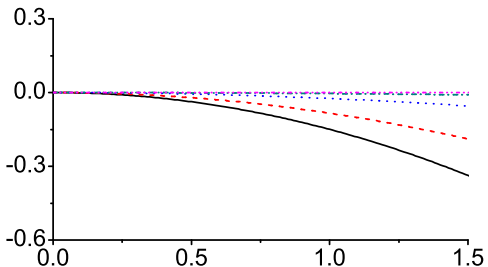

E

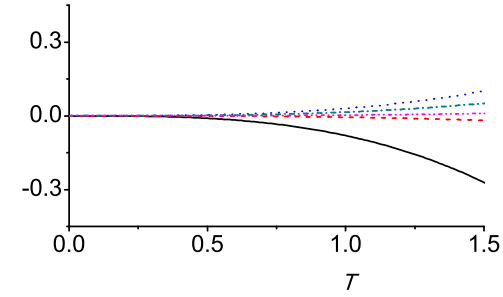

$A=0.7$
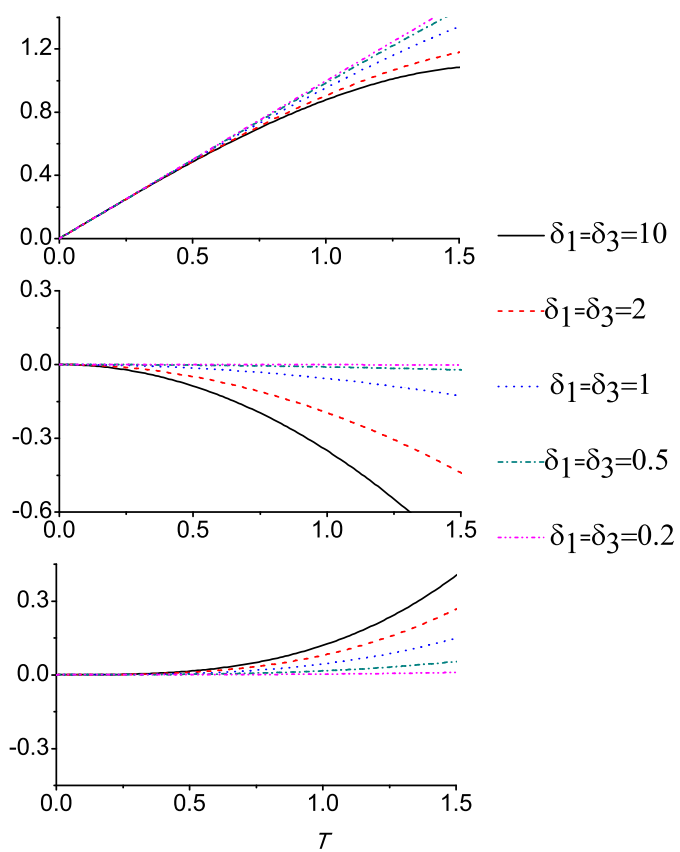

FIG. 5. Normalized amplitudes of bubbles and spikes of the middle interface versus normalized time $T=k v_{0} t$ for different finite-thicknesses $\delta_{1}=\delta_{3}$ $=10,2,1,0.5$, and 0.2 and Atwood numbers $A=0.3$ (left) and $A=0.7$ (right). The initial amplitude of the perturbation is $\hat{\varepsilon}=1 / 1000$. amplitudes of the bubble and the spike are nearly equal and grow faster when the thickness effect is taken into account.

Additionally, the thickness effect can be understood from some special cases. For the values of $B_{i}(i=1,2,3$, and 4) (see the Appendix) in the second harmonics, simple calculations provide the results that when $\delta_{1}=\delta_{3}, B_{1}=0$ and when $\delta_{1} \ll 1$ and $\delta_{3} \ll 1, B_{2}=B_{3}=-1$ and $B_{1}=B_{4}=0$, resulting in the amplitudes of the second harmonics tending to be zero. Similarly, when $\delta_{1} \ll 1$ and $\delta_{3} \ll 1$ taken into account, the amplitudes of the third harmonics tend to be zero. These trends can be found in Fig. 5 .

\section{CONCLUSION}

In classical RMI, the two semi-finite-thickness fluids are taken into account. However, in astronomical objects and engineering applications related to RMI, the thickness of the fluids is finite. Due to the importance of the harmonics in the evolution of the interface fingers (bubbles and spikes) in the weakly nonlinear stage, this paper mainly investigates the finite-thickness effect on the harmonics.

In the case of the initial single-mode cosine perturbation at the interface formed by the heavy fluid overlapping the light one in classical RMI, the amplitude of the second harmonics is always negative, resulting in the amplitude of the spikes larger than that of the bubbles, and the amplitude of the third harmonics is negative when the Atwood number is less than $1 / 2$. These factors influencing the amplitude evolution of the bubbles and spikes are revised by the thickness effect of the two fluids. The thickness effect on the harmonics is shown in two aspects: the phase and the size of the amplitude of the harmonics. It is found that the amplitude of the second harmonic will be positive when the thickness of the light fluid tends to be infinite and that of the heavy one is small. This positive amplitude of the second harmonics can make the amplitude of the bubbles be larger than that of the spikes (see Fig. 4).
Additionally, when the thickness of the two fluids is small, the amplitudes of the second and the third harmonics will tend to be zero, and the amplitude of the fundamental mode becomes larger, resulting in the amplitude of the bubble equal to that of the spike (see Fig. 5). These phenomena do not appear in classical RMI. Therefore, the thickness effect of the fluids plays an important role in the amplitude evolution of the harmonics at the weakly nonlinear stage of RMI.

\section{ACKNOWLEDGMENTS}

The authors sincerely thank the anonymous referee for the valuable comments and fruitful suggestions, leading to the present improved manuscript. This work was supported by the National Natural Science Foundation of China (Nos. 11502030, U1530261, 91852203, and 11472278), the National Key Research and Development Program of China (No. 2016YFA0401200) and Science Challenge Project (No. TZ2016001), the Innovation Fund of Fundamental Technology Institute of All Value In Creation (No. JCY2015A005), the Natural Science Foundation of Sichuan Province (Nos. 18ZA0260 and 2018JY0454), the Natural Science Foundation of Mianyang Normal University (Nos. HX2017007, HX201811, MYSY2017JC06, and MYSY2018T004), and the National High-Tech Inertial Confinement Fusion Committee.

\section{APPENDIX: FINITE-THICKNESS FACTORS IN THE MIDDLE INTERFACE}

The finite-thickness factors $B_{1}-B_{4}$ appeared in the second-order amplitude are

$$
\begin{aligned}
B_{1}= & 2 e^{-3 \delta_{1}}-3 e^{-2 \delta_{1}}+2 e^{-\delta_{1}}+2 e^{-3 \delta_{1}-4 \delta_{3}}-3 e^{-2 \delta_{1}-4 \delta_{3}} \\
& +2 e^{-\delta_{1}-4 \delta_{3}}-2 e^{-4 \delta_{1}-3 \delta_{3}}+3 e^{-4 \delta_{1}-2 \delta_{2}}-2 e^{-4 \delta_{1}-\delta_{3}} \\
& -2 e^{-3 \delta_{3}}+3 e^{-2 \delta_{3}}-2 e^{-\delta_{2}}
\end{aligned}
$$




$$
\begin{gathered}
B_{2}=e^{-4 \delta_{1}}-2 e^{-3 \delta_{1}}+3 e^{-2 \delta_{1}}-2 e^{-\delta_{1}}-2 e^{-3 \delta_{1}-4 \delta_{3}}+3 e^{-2 \delta_{1}-4 \delta_{3}}-2 e^{-\delta_{1}-4 \delta_{3}}-2 e^{-4 \delta_{1}-3 \delta_{3}}+3 e^{-4 \delta_{1}-2 \delta_{3}}-2 e^{-4 \delta_{1}-\delta_{3}} \\
+e^{4\left(-\delta_{1}-\delta_{3}\right)}+e^{-4 \delta_{3}}-2 e^{-3 \delta_{3}}+3 e^{-2 \delta_{3}}-2 e^{-\delta_{2}} \\
B_{3}=-e^{4\left(-\delta_{1}-\delta_{3}\right)} \\
B_{4}=e^{-4 \delta_{1}}-e^{-4 \delta_{3}}
\end{gathered}
$$

The finite-thickness factors $C_{0}-C_{9}$ appeared in the third-order amplitude of the fundamental mode are

$$
\begin{aligned}
& C_{0}=\left(e^{-2 \delta_{1}}+1\right)\left(e^{-2 \delta_{2}}+1\right)-1, \\
& C_{1}=\left(e^{-\delta_{1}-\delta_{3}}-1\right)^{2}\left(8 e^{-5 \delta_{1}}-16 e^{-4 \delta_{1}}+26 e^{-3 \delta_{1}}-14 e^{-2 \delta_{1}}+5 e^{-\delta_{1}}+5 e^{-4 \delta_{1}-5 \delta_{3}}-14 e^{-3 \delta_{1}-5 \delta_{2}}+26 e^{-2 \delta_{1}-5 \delta_{3}}-16 e^{-\delta_{1}-5 \delta_{3}}\right. \\
& +5 e^{-5 \delta_{1}-4 \delta_{3}}+8 e^{-3 \delta_{1}-4 \delta_{3}}-14 e^{-2 \delta_{1}-4 \delta_{3}}+20 e^{-\delta_{1}-4 \delta_{3}}-14 e^{-5 \delta_{1}-3 \delta_{3}}+8 e^{-4 \delta_{1}-3 \delta_{3}}+30 e^{-2 \delta_{1}-3 \delta_{3}}-14 e^{-\delta_{1}-3 \delta_{3}} \\
& +26 e^{-5 \delta_{1}-2 \delta_{3}}-14 e^{-4 \delta_{1}-2 \delta_{3}}+30 e^{-3 \delta_{1}-2 \delta_{3}}+8 e^{-\delta_{1}-2 \delta_{3}}-16 e^{-5 \delta_{1}-\delta_{3}}+20 e^{-4 \delta_{1}-\delta_{3}}-14 e^{-3 \delta_{1}-\delta_{3}}+8 e^{-2 \delta_{1}-\delta_{3}}-5 e^{-\delta_{1}-\delta_{3}} \\
& \left.-44 e^{2\left(-\delta_{1}-\delta_{3}\right)}-44 e^{3\left(-\delta_{1}-\delta_{3}\right)}-5 e^{4\left(-\delta_{1}-\delta_{3}\right)}+e^{5\left(-\delta_{1}-\delta_{3}\right)}+8 e^{-5 \delta_{3}}-16 e^{-4 \delta_{3}}+26 e^{-3 \delta_{3}}-14 e^{-2 \delta_{3}}+5 e^{-\delta_{3}}+1\right)-1, \\
& C_{2}=8 e^{-6 \delta_{1}}+e^{-5 \delta_{1}}+e^{-4 \delta_{1}}+e^{-3 \delta_{1}}+e^{-2 \delta_{1}}+8 e^{-\delta_{1}}+8 e^{-6 \delta_{1}-7 \delta_{3}}+e^{-5 \delta_{1}-7 \delta_{3}}+e^{-4 \delta_{1}-7 \delta_{3}}+e^{-3 \delta_{1}-7 \delta_{3}}+e^{-2 \delta_{1}-7 \delta_{3}}+8 e^{-\delta_{1}-7 \delta_{3}} \\
& -8 e^{-7 \delta_{1}-6 \delta_{2}}-7 e^{-5 \delta_{1}-6 \delta_{3}}-7 e^{-4 \delta_{1}-6 \delta_{3}}-7 e^{-3 \delta_{1}-6 \delta_{3}}-7 e^{-2 \delta_{1}-6 \delta_{3}}-e^{-7 \delta_{1}-5 \delta_{3}}+7 e^{-6 \delta_{1}-5 \delta_{3}}+7 e^{-\delta_{1}-5 \delta_{3}}-e^{-7 \delta_{1}-4 \delta_{3}} \\
& +7 e^{-6 \delta_{1}-4 \delta_{3}}+7 e^{-\delta_{1}-4 \delta_{2}}-e^{-7 \delta_{1}-3 \delta_{3}}+7 e^{-6 \delta_{1}-3 \delta_{3}}+7 e^{-\delta_{1}-3 \delta_{3}}-e^{-7 \delta_{1}-2 \delta_{3}}+7 e^{-6 \delta_{1}-2 \delta_{3}}+7 e^{-\delta_{1}-2 \delta_{3}}-8 e^{-7 \delta_{1}-\delta_{3}} \\
& -7 e^{-5 \delta_{1}-\delta_{3}}-7 e^{-4 \delta_{1}-\delta_{3}}-7 e^{-3 \delta_{1}-\delta_{3}}-7 e^{-2 \delta_{1}-\delta_{3}}-8 e^{-6 \delta_{3}}-e^{-5 \delta_{3}}-e^{-4 \delta_{3}}-e^{-3 \delta_{3}}-e^{-2 \delta_{3}}-8 e^{-\delta_{3}}, \\
& C_{3}=5 e^{-7 \delta_{1}}-3 e^{-6 \delta_{1}}-4 e^{-5 \delta_{1}}+20 e^{-4 \delta_{1}}-22 e^{-3 \delta_{1}}+18 e^{-2 \delta_{1}}-8 e^{-\delta_{1}}-8 e^{-6 \delta_{1}-7 \delta_{3}}+18 e^{-5 \delta_{1}-7 \delta_{3}}-22 e^{-4 \delta_{1}-7 \delta_{3}} \\
& +20 e^{-3 \delta_{1}-7 \delta_{3}}-4 e^{-2 \delta_{1}-7 \delta_{3}}-3 e^{-\delta_{1}-7 \delta_{3}}-8 e^{-7 \delta_{1}-6 \delta_{3}}-2 e^{-5 \delta_{1}-6 \delta_{3}}-18 e^{-4 \delta_{1}-6 \delta_{3}}+28 e^{-3 \delta_{1}-6 \delta_{3}}-20 e^{-2 \delta_{1}-6 \delta_{3}} \\
& +5 e^{-\delta_{1}-6 \delta_{3}}+18 e^{-7 \delta_{1}-5 \delta_{3}}-2 e^{-6 \delta_{1}-5 \delta_{3}}-16 e^{-4 \delta_{1}-5 \delta_{3}}+3 e^{-3 \delta_{1}-5 \delta_{3}}-9 e^{-2 \delta_{1}-5 \delta_{3}}-20 e^{-\delta_{1}-5 \delta_{2}}-22 e^{-7 \delta_{1}-4 \delta_{3}} \\
& -18 e^{-6 \delta_{1}-4 \delta_{3}}-16 e^{-5 \delta_{1}-4 \delta_{3}}+47 e^{-3 \delta_{1}-4 \delta_{3}}+3 e^{-2 \delta_{1}-4 \delta_{3}}+28 e^{-\delta_{1}-4 \delta_{3}}+20 e^{-7 \delta_{1}-3 \delta_{3}}+28 e^{-6 \delta_{1}-3 \delta_{3}}+3 e^{-5 \delta_{1}-3 \delta_{3}} \\
& +47 e^{-4 \delta_{1}-3 \delta_{3}}-16 e^{-2 \delta_{1}-3 \delta_{3}}-18 e^{-\delta_{1}-3 \delta_{3}}-4 e^{-7 \delta_{1}-2 \delta_{3}}-20 e^{-6 \delta_{1}-2 \delta_{3}}-9 e^{-5 \delta_{1}-2 \delta_{3}}+3 e^{-4 \delta_{1}-2 \delta_{2}}-16 e^{-3 \delta_{1}-2 \delta_{3}} \\
& -2 e^{-\delta_{1}-2 \delta_{3}}-3 e^{-7 \delta_{1}-\delta_{3}}+5 e^{-6 \delta_{1}-\delta_{3}}-20 e^{-5 \delta_{1}-\delta_{3}}+28 e^{-4 \delta_{1}-\delta_{3}}-18 e^{-3 \delta_{1}-\delta_{3}}-2 e^{-2 \delta_{1}-\delta_{3}}-4 e^{-\delta_{1}-\delta_{3}}+36 e^{2\left(-\delta_{1}-\delta_{2}\right)} \\
& -36 e^{3\left(-\delta_{1}-\delta_{3}\right)}-36 e^{4\left(-\delta_{1}-\delta_{3}\right)}+36 e^{5\left(-\delta_{1}-\delta_{3}\right)}-4 e^{6\left(-\delta_{1}-\delta_{3}\right)}+4 e^{7\left(-\delta_{1}-\delta_{3}\right)}+5 e^{-7 \delta_{3}}-3 e^{-6 \delta_{3}}-4 e^{-5 \delta_{3}}+20 e^{-4 \delta_{3}}-22 e^{-3 \delta_{3}} \\
& +18 e^{-2 \delta_{3}}-8 e^{-\delta_{3}}, \\
& \begin{aligned}
C_{4}= & \left(-1+e^{-\delta_{1}-\delta_{3}}\right)^{2}\left(1-4 e^{-6 \delta_{1}}+2 e^{-5 \delta_{1}}-6 e^{-4 \delta_{1}}+7 e^{-3 \delta_{1}}-5 e^{-2 \delta_{1}}+e^{-\delta_{1}}+e^{-6 \delta_{1}-7 \delta_{3}}-5 e^{-5 \delta_{1}-7 \delta_{3}}+7 e^{-4 \delta_{1}-7 \delta_{3}}\right. \\
& -6 e^{-3 \delta_{1}-7 \delta_{3}}+2 e^{-2 \delta_{1}-7 \delta_{3}}-4 e^{-\delta_{1}-7 \delta_{3}}+e^{-7 \delta_{1}-6 \delta_{3}}+e^{-5 \delta_{1}-6 \delta_{2}}+e^{-4 \delta_{1}-6 \delta_{3}}+16 e^{-3 \delta_{1}-6 \delta_{3}}-2 e^{-2 \delta_{1}-6 \delta_{3}}+4 e^{-\delta_{1}-6 \delta_{3}} \\
& -5 e^{-7 \delta_{1}-5 \delta_{3}}+e^{-6 \delta_{1}-5 \delta_{3}}+5 e^{-4 \delta_{1}-5 \delta_{3}}-16 e^{-3 \delta_{1}-5 \delta_{3}}+18 e^{-2 \delta_{1}-5 \delta_{3}}-2 e^{-\delta_{1}-5 \delta_{3}}+7 e^{-7 \delta_{1}-4 \delta_{3}}+e^{-6 \delta_{1}-4 \delta_{3}}+5 e^{-5 \delta_{1}-4 \delta_{2}} \\
& +10 e^{-3 \delta_{1}-4 \delta_{3}}-16 e^{-2 \delta_{1}-4 \delta_{3}}+16 e^{-\delta_{1}-4 \delta_{3}}-6 e^{-7 \delta_{1}-3 \delta_{3}}+16 e^{-6 \delta_{1}-3 \delta_{3}}-16 e^{-5 \delta_{1}-3 \delta_{3}}+10 e^{-4 \delta_{1}-3 \delta_{3}}+5 e^{-2 \delta_{1}-3 \delta_{3}} \\
& +e^{-\delta_{1}-3 \delta_{3}}+2 e^{-7 \delta_{1}-2 \delta_{3}}-2 e^{-6 \delta_{1}-2 \delta_{3}}+18 e^{-5 \delta_{1}-2 \delta_{3}}-16 e^{-4 \delta_{1}-2 \delta_{2}}+5 e^{-3 \delta_{1}-2 \delta_{3}}+e^{-\delta_{1}-2 \delta_{3}}-4 e^{-7 \delta_{1}-\delta_{3}}+4 e^{-6 \delta_{1}-\delta_{3}} \\
& -2 e^{-5 \delta_{1}-\delta_{3}}+16 e^{-4 \delta_{1}-\delta_{3}}+e^{-3 \delta_{1}-\delta_{2}}+e^{-2 \delta_{1}-\delta_{3}}+3 e^{-\delta_{1}-\delta_{3}}-23 e^{2\left(-\delta_{1}-\delta_{2}\right)}-13 e^{3\left(-\delta_{1}-\delta_{3}\right)}-13 e^{4\left(-\delta_{1}-\delta_{3}\right)}-23 e^{5\left(-\delta_{1}-\delta_{3}\right)} \\
& \left.+3 e^{6\left(-\delta_{1}-\delta_{3}\right)}+e^{7\left(-\delta_{1}-\delta_{3}\right)}-4 e^{-6 \delta_{3}}+2 e^{-5 \delta_{3}}-6 e^{-4 \delta_{3}}+7 e^{-3 \delta_{3}}-5 e^{-2 \delta_{3}}+e^{-\delta_{3}}\right)-1
\end{aligned} \\
& C_{5}=-\left(e^{-3 \delta_{1}}+e^{-2 \delta_{1}}+e^{-\delta_{1}}+1\right)\left(-5 e^{-4 \delta_{1}}+8 e^{-3 \delta_{1}}-5 e^{-2 \delta_{1}}-5 e^{-4 \delta_{1}-6 \delta_{3}}+8 e^{-3 \delta_{1}-6 \delta_{3}}-5 e^{-2 \delta_{1}-6 \delta_{3}}+5 e^{-6 \delta_{1}-4 \delta_{3}}\right. \\
& +8 e^{-3 \delta_{1}-4 \delta_{3}}-8 e^{-6 \delta_{1}-3 \delta_{3}}-8 e^{-4 \delta_{1}-3 \delta_{3}}-8 e^{-2 \delta_{1}-3 \delta_{3}}+5 e^{-6 \delta_{1}-2 \delta_{2}}+8 e^{-3 \delta_{1}-2 \delta_{3}}+5 e^{-4 \delta_{3}}-8 e^{-3 \delta_{3}} \\
& \left.+5 e^{-2 \delta_{3}}\right)\left(e^{-3 \delta_{3}}+e^{-2 \delta_{3}}+e^{-\delta_{3}}+1\right),
\end{aligned}
$$




$$
\begin{aligned}
& C_{6}=e^{-9 \delta_{1}}+e^{-8 \delta_{1}}-3 e^{-7 \delta_{1}}+9 e^{-6 \delta_{1}}-2 e^{-5 \delta_{1}}+6 e^{-4 \delta_{1}}-2 e^{-3 \delta_{1}}+2 e^{-2 \delta_{1}}+2 e^{-7 \delta_{1}-9 \delta_{3}}-2 e^{-6 \delta_{1}-9 \delta_{2}}+6 e^{-5 \delta_{1}-9 \delta_{3}} \\
& -2 e^{-4 \delta_{1}-9 \delta_{3}}+9 e^{-3 \delta_{1}-9 \delta_{3}}-3 e^{-2 \delta_{1}-9 \delta_{3}}+e^{-\delta_{1}-9 \delta_{3}}-2 e^{-7 \delta_{1}-8 \delta_{3}}-6 e^{-6 \delta_{1}-8 \delta_{3}}-2 e^{-5 \delta_{1}-8 \delta_{3}}-10 e^{-4 \delta_{1}-8 \delta_{3}}+5 e^{-3 \delta_{1}-8 \delta_{3}} \\
& -7 e^{-2 \delta_{1}-8 \delta_{3}}+e^{-\delta_{1}-8 \delta_{3}}+2 e^{-9 \delta_{1}-7 \delta_{3}}-2 e^{-8 \delta_{1}-7 \delta_{3}}-4 e^{-6 \delta_{1}-7 \delta_{3}}+13 e^{-5 \delta_{1}-7 \delta_{3}}-3 e^{-4 \delta_{1}-7 \delta_{3}}+4 e^{-3 \delta_{1}-7 \delta_{3}}-12 e^{-2 \delta_{1}-7 \delta_{3}} \\
& -7 e^{-\delta_{1}-7 \delta_{3}}-2 e^{-9 \delta_{1}-6 \delta_{3}}-6 e^{-8 \delta_{1}-6 \delta_{3}}-4 e^{-7 \delta_{1}-6 \delta_{3}}+5 e^{-5 \delta_{1}-6 \delta_{3}}-11 e^{-4 \delta_{1}-6 \delta_{3}}+20 e^{-3 \delta_{1}-6 \delta_{2}}+4 e^{-2 \delta_{1}-6 \delta_{3}}+5 e^{-\delta_{1}-6 \delta_{3}} \\
& +6 e^{-9 \delta_{1}-5 \delta_{3}}-2 e^{-8 \delta_{1}-5 \delta_{3}}+13 e^{-7 \delta_{1}-5 \delta_{3}}+5 e^{-6 \delta_{1}-5 \delta_{3}}+6 e^{-4 \delta_{1}-5 \delta_{3}}-11 e^{-3 \delta_{1}-5 \delta_{3}}-3 e^{-2 \delta_{1}-5 \delta_{3}}-10 e^{-\delta_{1}-5 \delta_{3}}-2 e^{-9 \delta_{1}-4 \delta_{3}} \\
& -10 e^{-8 \delta_{1}-4 \delta_{3}}-3 e^{-7 \delta_{1}-4 \delta_{2}}-11 e^{-6 \delta_{1}-4 \delta_{3}}+6 e^{-5 \delta_{1}-4 \delta_{3}}+5 e^{-3 \delta_{1}-4 \delta_{3}}+13 e^{-2 \delta_{1}-4 \delta_{3}}-2 e^{-\delta_{1}-4 \delta_{3}}+9 e^{-9 \delta_{1}-3 \delta_{3}}+5 e^{-8 \delta_{1}-3 \delta_{3}} \\
& +4 e^{-7 \delta_{1}-3 \delta_{3}}+20 e^{-6 \delta_{1}-3 \delta_{3}}-11 e^{-5 \delta_{1}-3 \delta_{3}}+5 e^{-4 \delta_{1}-3 \delta_{3}}-4 e^{-2 \delta_{1}-3 \delta_{3}}-6 e^{-\delta_{1}-3 \delta_{2}}-3 e^{-9 \delta_{1}-2 \delta_{3}}-7 e^{-8 \delta_{1}-2 \delta_{3}}-12 e^{-7 \delta_{1}-2 \delta_{3}} \\
& +4 e^{-6 \delta_{1}-2 \delta_{3}}-3 e^{-5 \delta_{1}-2 \delta_{3}}+13 e^{-4 \delta_{1}-2 \delta_{3}}-4 e^{-3 \delta_{1}-2 \delta_{3}}-2 e^{-\delta_{1}-2 \delta_{3}}+e^{-9 \delta_{1}-\delta_{3}}+e^{-8 \delta_{1}-\delta_{2}}-7 e^{-7 \delta_{1}-\delta_{3}}+5 e^{-6 \delta_{1}-\delta_{3}} \\
& -10 e^{-5 \delta_{1}-\delta_{3}}-2 e^{-4 \delta_{1}-\delta_{3}}-6 e^{-3 \delta_{1}-\delta_{3}}-2 e^{-2 \delta_{1}-\delta_{3}}+12 e^{2\left(-\delta_{1}-\delta_{3}\right)}-20 e^{3\left(-\delta_{1}-\delta_{3}\right)}+6 e^{4\left(-\delta_{1}-\delta_{2}\right)}+6 e^{5\left(-\delta_{1}-\delta_{3}\right)} \\
& -20 e^{6\left(-\delta_{1}-\delta_{3}\right)}+12 e^{7\left(-\delta_{1}-\delta_{3}\right)}+e^{-9 \delta_{3}}+e^{-8 \delta_{3}}-3 e^{-7 \delta_{3}}+9 e^{-6 \delta_{3}}-2 e^{-5 \delta_{3}}+6 e^{-4 \delta_{3}}-2 e^{-3 \delta_{3}}+2 e^{-2 \delta_{3}}, \\
& C_{7}=\left(e^{-3 \delta_{1}}+e^{-2 \delta_{1}}+e^{-\delta_{1}}+1\right)\left(-e^{2\left(-\delta_{1}-\delta_{2}\right)}-e^{4\left(-\delta_{1}-\delta_{3}\right)}+e^{6\left(-\delta_{1}-\delta_{3}\right)}+1\right)\left(e^{-3 \delta_{3}}+e^{-2 \delta_{3}}+e^{-\delta_{3}}+1\right)-1, \\
& C_{8}=-\left(e^{-2 \delta_{1}}+1\right)^{2}\left(e^{-\delta_{1}}+1\right)\left(e^{-2 \delta_{3}}+1\right)^{2}\left(-e^{-2 \delta_{1}}-e^{-2 \delta_{1}-4 \delta_{3}}+e^{-4 \delta_{1}-2 \delta_{3}}+e^{-2 \delta_{2}}\right)\left(e^{-\delta_{3}}+1\right), \\
& C_{9}=\left(e^{-3 \delta_{1}}+e^{-2 \delta_{1}}+e^{-\delta_{1}}+1\right)\left(e^{-2 \delta_{1}}-e^{-2 \delta_{2}}\right)^{2}\left(e^{-2 \delta_{1}}+e^{-2 \delta_{3}}\right)\left(e^{-3 \delta_{3}}+e^{-2 \delta_{2}}+e^{-\delta_{3}}+1\right) .
\end{aligned}
$$

The finite-thickness factors $F_{1}-F_{9}$ appeared in the third-order amplitude of the third harmonics are

$$
\begin{aligned}
& F_{1}=1-\left(-1+e^{-\delta_{1}-\delta_{3}}\right)^{2}\left(1-8 e^{-9 \delta_{1}}+12 e^{-8 \delta_{1}}-8 e^{-7 \delta_{1}}-8 e^{-6 \delta_{1}}+26 e^{-5 \delta_{1}}-34 e^{-4 \delta_{1}}+20 e^{-3 \delta_{1}}-4 e^{-2 \delta_{1}}-3 e^{-\delta_{1}}\right. \\
& -3 e^{-8 \delta_{1}-9 \delta_{3}}-4 e^{-7 \delta_{1}-9 \delta_{3}}+20 e^{-6 \delta_{1}-9 \delta_{3}}-34 e^{-5 \delta_{1}-9 \delta_{3}}+26 e^{-4 \delta_{1}-9 \delta_{3}}-8 e^{-3 \delta_{1}-9 \delta_{3}}-8 e^{-2 \delta_{1}-9 \delta_{3}}+12 e^{-\delta_{1}-9 \delta_{3}} \\
& -3 e^{-9 \delta_{1}-8 \delta_{3}}-14 e^{-7 \delta_{1}-8 \delta_{3}}+8 e^{-6 \delta_{1}-8 \delta_{3}}+26 e^{-5 \delta_{1}-8 \delta_{2}}-46 e^{-4 \delta_{1}-8 \delta_{3}}+36 e^{-3 \delta_{1}-8 \delta_{3}}-8 e^{-2 \delta_{1}-8 \delta_{3}}-8 e^{-\delta_{1}-8 \delta_{3}} \\
& -4 e^{-9 \delta_{1}-7 \delta_{3}}-14 e^{-8 \delta_{1}-7 \delta_{3}}-25 e^{-6 \delta_{1}-7 \delta_{3}}+6 e^{-5 \delta_{1}-7 \delta_{3}}+26 e^{-4 \delta_{1}-7 \delta_{3}}-60 e^{-3 \delta_{1}-7 \delta_{3}}+36 e^{-2 \delta_{1}-7 \delta_{3}}-8 e^{-\delta_{1}-7 \delta_{3}} \\
& +20 e^{-9 \delta_{1}-6 \delta_{3}}+8 e^{-8 \delta_{1}-6 \delta_{3}}-25 e^{-7 \delta_{1}-6 \delta_{3}}-30 e^{-5 \delta_{1}-6 \delta_{2}}+18 e^{-4 \delta_{1}-6 \delta_{3}}+48 e^{-3 \delta_{1}-6 \delta_{3}}-60 e^{-2 \delta_{1}-6 \delta_{3}}+36 e^{-\delta_{1}-6 \delta_{3}} \\
& -34 e^{-9 \delta_{1}-5 \delta_{3}}+26 e^{-8 \delta_{1}-5 \delta_{3}}+6 e^{-7 \delta_{1}-5 \delta_{3}}-30 e^{-6 \delta_{1}-5 \delta_{3}}-56 e^{-4 \delta_{1}-5 \delta_{3}}+18 e^{-3 \delta_{1}-5 \delta_{3}}+26 e^{-2 \delta_{1}-5 \delta_{3}}-46 e^{-\delta_{1}-5 \delta_{3}} \\
& +26 e^{-9 \delta_{1}-4 \delta_{3}}-46 e^{-8 \delta_{1}-4 \delta_{3}}+26 e^{-7 \delta_{1}-4 \delta_{3}}+18 e^{-6 \delta_{1}-4 \delta_{2}}-56 e^{-5 \delta_{1}-4 \delta_{3}}-30 e^{-3 \delta_{1}-4 \delta_{3}}+6 e^{-2 \delta_{1}-4 \delta_{3}}+26 e^{-\delta_{1}-4 \delta_{3}} \\
& -8 e^{-9 \delta_{1}-3 \delta_{3}}+36 e^{-8 \delta_{1}-3 \delta_{3}}-60 e^{-7 \delta_{1}-3 \delta_{3}}+48 e^{-6 \delta_{1}-3 \delta_{3}}+18 e^{-5 \delta_{1}-3 \delta_{3}}-30 e^{-4 \delta_{1}-3 \delta_{3}}-25 e^{-2 \delta_{1}-3 \delta_{3}}+8 e^{-\delta_{1}-3 \delta_{3}} \\
& -8 e^{-9 \delta_{1}-2 \delta_{3}}-8 e^{-8 \delta_{1}-2 \delta_{3}}+36 e^{-7 \delta_{1}-2 \delta_{3}}-60 e^{-6 \delta_{1}-2 \delta_{2}}+26 e^{-5 \delta_{1}-2 \delta_{3}}+6 e^{-4 \delta_{1}-2 \delta_{3}}-25 e^{-3 \delta_{1}-2 \delta_{3}}-14 e^{-\delta_{1}-2 \delta_{3}} \\
& +12 e^{-9 \delta_{1}-\delta_{3}}-8 e^{-8 \delta_{1}-\delta_{3}}-8 e^{-7 \delta_{1}-\delta_{3}}+36 e^{-6 \delta_{1}-\delta_{3}}-46 e^{-5 \delta_{1}-\delta_{3}}+26 e^{-4 \delta_{1}-\delta_{2}}+8 e^{-3 \delta_{1}-\delta_{3}}-14 e^{-2 \delta_{1}-\delta_{3}}+11 e^{-\delta_{1}-\delta_{3}} \\
& +21 e^{2\left(-\delta_{1}-\delta_{3}\right)}+31 e^{3\left(-\delta_{1}-\delta_{3}\right)}+48 e^{4\left(-\delta_{1}-\delta_{3}\right)}+48 e^{5\left(-\delta_{1}-\delta_{3}\right)}+31 e^{6\left(-\delta_{1}-\delta_{2}\right)}+21 e^{7\left(-\delta_{1}-\delta_{3}\right)}+11 e^{8\left(-\delta_{1}-\delta_{3}\right)}+e^{9\left(-\delta_{1}-\delta_{3}\right)} \\
& \left.-8 e^{-9 \delta_{3}}+12 e^{-8 \delta_{3}}-8 e^{-7 \delta_{3}}-8 e^{-6 \delta_{3}}+26 e^{-5 \delta_{3}}-34 e^{-4 \delta_{3}}+20 e^{-3 \delta_{3}}-4 e^{-2 \delta_{3}}-3 e^{-\delta_{2}}\right),
\end{aligned}
$$

$$
\begin{aligned}
F_{2}= & \left(1+e^{-\delta_{1}}\right)\left(1+e^{-\delta_{3}}\right)\left(-8 e^{-9 \delta_{1}}+24 e^{-8 \delta_{1}}-52 e^{-7 \delta_{1}}+81 e^{-6 \delta_{1}}-96 e^{-5 \delta_{1}}+81 e^{-4 \delta_{1}}-52 e^{-3 \delta_{1}}+24 e^{-2 \delta_{1}}-8 e^{-\delta_{1}}\right. \\
& -8 e^{-9 \delta_{1}-10 \delta_{3}}+24 e^{-8 \delta_{1}-10 \delta_{2}}-52 e^{-7 \delta_{1}-10 \delta_{3}}+81 e^{-6 \delta_{1}-10 \delta_{3}}+81 e^{-4 \delta_{1}-10 \delta_{2}}-52 e^{-3 \delta_{1}-10 \delta_{3}}-8 e^{-\delta_{1}-10 \delta_{3}}+8 e^{-10 \delta_{1}-9 \delta_{3}} \\
& +8 e^{-6 \delta_{1}-9 \delta_{3}}+8 e^{-4 \delta_{1}-9 \delta_{3}}-24 e^{-10 \delta_{1}-8 \delta_{3}}-24 e^{-6 \delta_{1}-8 \delta_{3}}-24 e^{-4 \delta_{1}-8 \delta_{3}}+52 e^{-10 \delta_{1}-7 \delta_{3}}+52 e^{-6 \delta_{1}-7 \delta_{3}} \\
& +52 e^{-4 \delta_{1}-7 \delta_{3}}-81 e^{-10 \delta_{1}-6 \delta_{3}}-8 e^{-9 \delta_{1}-6 \delta_{3}}+24 e^{-8 \delta_{1}-6 \delta_{3}}-52 e^{-7 \delta_{1}-6 \delta_{3}}-96 e^{-5 \delta_{1}-6 \delta_{3}}-52 e^{-3 \delta_{1}-6 \delta_{3}}+24 e^{-2 \delta_{1}-6 \delta_{3}} \\
& -8 e^{-\delta_{1}-6 \delta_{2}}+96 e^{-6 \delta_{1}-5 \delta_{3}}+96 e^{-4 \delta_{1}-5 \delta_{3}}+24 e^{2\left(-\delta_{1}-5 \delta_{2}\right)}-81 e^{-10 \delta_{1}-4 \delta_{3}}-8 e^{-9 \delta_{1}-4 \delta_{3}}+24 e^{-8 \delta_{1}-4 \delta_{2}}-52 e^{-7 \delta_{1}-4 \delta_{3}} \\
& -96 e^{-5 \delta_{1}-4 \delta_{3}}-52 e^{-3 \delta_{1}-4 \delta_{3}}+24 e^{-2 \delta_{1}-4 \delta_{3}}-8 e^{-\delta_{1}-4 \delta_{3}}+52 e^{-10 \delta_{1}-3 \delta_{3}}+52 e^{-6 \delta_{1}-3 \delta_{3}}+52 e^{-4 \delta_{1}-3 \delta_{3}}-24 e^{-6 \delta_{1}-2 \delta_{3}} \\
& -24 e^{-4 \delta_{1}-2 \delta_{3}}-96 e^{5\left(-\delta_{1}-2 \delta_{3}\right)}+8 e^{-10 \delta_{1}-\delta_{3}}+8 e^{-6 \delta_{1}-\delta_{3}}-24 e^{2\left(-5 \delta_{1}-\delta_{3}\right)}+8 e^{-4 \delta_{1}-\delta_{3}}+96 e^{5\left(-2 \delta_{1}-\delta_{3}\right)}+8 e^{-9 \delta_{3}}-24 e^{-8 \delta_{3}} \\
& \left.+52 e^{-7 \delta_{3}}-81 e^{-6 \delta_{3}}+96 e^{-5 \delta_{3}}-81 e^{-4 \delta_{3}}+52 e^{-3 \delta_{3}}-24 e^{-2 \delta_{3}}+8 e^{-\delta_{3}}\right),
\end{aligned}
$$




$$
\begin{aligned}
& F_{3}=3 e^{-11 \delta_{1}}-5 e^{-10 \delta_{1}}+8 e^{-9 \delta_{1}}-16 e^{-8 \delta_{1}}+24 e^{-7 \delta_{1}}-20 e^{-6 \delta_{1}}+14 e^{-5 \delta_{1}}-2 e^{-4 \delta_{1}}-8 e^{-3 \delta_{1}}+12 e^{-2 \delta_{1}}-8 e^{-\delta_{1}} \\
& -8 e^{-10 \delta_{1}-11 \delta_{3}}+12 e^{-9 \delta_{1}-11 \delta_{3}}-8 e^{-8 \delta_{1}-11 \delta_{3}}-2 e^{-7 \delta_{1}-11 \delta_{3}}+14 e^{-6 \delta_{1}-11 \delta_{3}}-20 e^{-5 \delta_{1}-11 \delta_{3}}+24 e^{-4 \delta_{1}-11 \delta_{3}}-16 e^{-3 \delta_{1}-11 \delta_{3}} \\
& +8 e^{-2 \delta_{1}-11 \delta_{3}}-5 e^{-\delta_{1}-11 \delta_{3}}-8 e^{-11 \delta_{1}-10 \delta_{3}}+8 e^{-9 \delta_{1}-10 \delta_{2}}-12 e^{-8 \delta_{1}-10 \delta_{3}}+10 e^{-7 \delta_{1}-10 \delta_{3}}+2 e^{-6 \delta_{1}-10 \delta_{2}}+32 e^{-4 \delta_{1}-10 \delta_{3}} \\
& -20 e^{-3 \delta_{1}-10 \delta_{3}}+3 e^{-\delta_{1}-10 \delta_{3}}+12 e^{-11 \delta_{1}-9 \delta_{3}}+8 e^{-10 \delta_{1}-9 \delta_{3}}+2 e^{-7 \delta_{1}-9 \delta_{3}}+10 e^{-6 \delta_{1}-9 \delta_{3}}+14 e^{-5 \delta_{1}-9 \delta_{3}}+6 e^{-4 \delta_{1}-9 \delta_{3}} \\
& +4 e^{-\delta_{1}-9 \delta_{3}}-8 e^{-11 \delta_{1}-8 \delta_{3}}-12 e^{-10 \delta_{1}-8 \delta_{3}}-22 e^{-7 \delta_{1}-8 \delta_{3}}-14 e^{-6 \delta_{1}-8 \delta_{3}}-6 e^{-5 \delta_{1}-8 \delta_{3}}-14 e^{-4 \delta_{1}-8 \delta_{2}}-20 e^{-\delta_{1}-8 \delta_{3}} \\
& -2 e^{-11 \delta_{1}-7 \delta_{3}}+10 e^{-10 \delta_{1}-7 \delta_{3}}+2 e^{-9 \delta_{1}-7 \delta_{3}}-22 e^{-8 \delta_{1}-7 \delta_{3}}-4 e^{-6 \delta_{1}-7 \delta_{3}}+5 e^{-5 \delta_{1}-7 \delta_{3}}+17 e^{-4 \delta_{1}-7 \delta_{3}}-14 e^{-3 \delta_{1}-7 \delta_{3}} \\
& +6 e^{-2 \delta_{1}-7 \delta_{3}}+32 e^{-\delta_{1}-7 \delta_{3}}+14 e^{-11 \delta_{1}-6 \delta_{3}}+2 e^{-10 \delta_{1}-6 \delta_{3}}+10 e^{-9 \delta_{1}-6 \delta_{3}}-14 e^{-8 \delta_{1}-6 \delta_{3}}-4 e^{-7 \delta_{1}-6 \delta_{2}}+25 e^{-5 \delta_{1}-6 \delta_{3}} \\
& +5 e^{-4 \delta_{1}-6 \delta_{3}}-6 e^{-3 \delta_{1}-6 \delta_{3}}+14 e^{-2 \delta_{1}-6 \delta_{3}}-36 e^{-\delta_{1}-6 \delta_{3}}-20 e^{-11 \delta_{1}-5 \delta_{3}}+14 e^{-9 \delta_{1}-5 \delta_{3}}-6 e^{-8 \delta_{1}-5 \delta_{3}}+5 e^{-7 \delta_{1}-5 \delta_{3}} \\
& +25 e^{-6 \delta_{1}-5 \delta_{3}}-4 e^{-4 \delta_{1}-5 \delta_{3}}-14 e^{-3 \delta_{1}-5 \delta_{3}}+10 e^{-2 \delta_{1}-5 \delta_{3}}+2 e^{-\delta_{1}-5 \delta_{3}}+4 e^{2\left(-\delta_{1}-5 \delta_{3}\right)}+24 e^{-11 \delta_{1}-4 \delta_{3}}+32 e^{-10 \delta_{1}-4 \delta_{3}} \\
& +6 e^{-9 \delta_{1}-4 \delta_{3}}-14 e^{-8 \delta_{1}-4 \delta_{3}}+17 e^{-7 \delta_{1}-4 \delta_{3}}+5 e^{-6 \delta_{1}-4 \delta_{3}}-4 e^{-5 \delta_{1}-4 \delta_{2}}-22 e^{-3 \delta_{1}-4 \delta_{3}}+2 e^{-2 \delta_{1}-4 \delta_{3}}+10 e^{-\delta_{1}-4 \delta_{3}} \\
& -16 e^{-11 \delta_{1}-3 \delta_{3}}-20 e^{-10 \delta_{1}-3 \delta_{3}}-14 e^{-7 \delta_{1}-3 \delta_{3}}-6 e^{-6 \delta_{1}-3 \delta_{3}}-14 e^{-5 \delta_{1}-3 \delta_{3}}-22 e^{-4 \delta_{1}-3 \delta_{3}}-12 e^{-\delta_{1}-3 \delta_{3}}+8 e^{-11 \delta_{1}-2 \delta_{3}} \\
& +6 e^{-7 \delta_{1}-2 \delta_{3}}+14 e^{-6 \delta_{1}-2 \delta_{3}}+10 e^{-5 \delta_{1}-2 \delta_{3}}+2 e^{-4 \delta_{1}-2 \delta_{3}}+8 e^{-\delta_{1}-2 \delta_{2}}-36 e^{5\left(-\delta_{1}-2 \delta_{3}\right)}-5 e^{-11 \delta_{1}-\delta_{3}}+3 e^{-10 \delta_{1}-\delta_{3}} \\
& +4 e^{-9 \delta_{1}-\delta_{3}}-20 e^{-8 \delta_{1}-\delta_{3}}+32 e^{-7 \delta_{1}-\delta_{2}}-36 e^{-6 \delta_{1}-\delta_{3}}+2 e^{-5 \delta_{1}-\delta_{3}}+4 e^{2\left(-5 \delta_{1}-\delta_{2}\right)}+10 e^{-4 \delta_{1}-\delta_{3}}-12 e^{-3 \delta_{1}-\delta_{3}}+8 e^{-2 \delta_{1}-\delta_{3}} \\
& -36 e^{5\left(-2 \delta_{1}-\delta_{3}\right)}-4 e^{-\delta_{1}-\delta_{3}}+68 e^{4\left(-\delta_{1}-\delta_{2}\right)}-44 e^{5\left(-\delta_{1}-\delta_{3}\right)}-44 e^{6\left(-\delta_{1}-\delta_{3}\right)}+68 e^{7\left(-\delta_{1}-\delta_{3}\right)}-4 e^{10\left(-\delta_{1}-\delta_{3}\right)}+4 e^{11\left(-\delta_{1}-\delta_{3}\right)} \\
& +3 e^{-11 \delta_{3}}-5 e^{-10 \delta_{3}}+8 e^{-9 \delta_{3}}-16 e^{-8 \delta_{3}}+24 e^{-7 \delta_{3}}-20 e^{-6 \delta_{3}}+14 e^{-5 \delta_{3}}-2 e^{-4 \delta_{3}}-8 e^{-3 \delta_{3}}+12 e^{-2 \delta_{3}}-8 e^{-\delta_{3}},
\end{aligned}
$$

$F_{4}=\left(-1+e^{-\delta_{1}-\delta_{3}}\right)^{2}\left(1-2 e^{-7 \delta_{1}}-2 e^{-6 \delta_{1}}-6 e^{-5 \delta_{1}}+6 e^{-4 \delta_{1}}-3 e^{-3 \delta_{1}}+5 e^{-2 \delta_{1}}-3 e^{-\delta_{1}}-3 e^{-10 \delta_{1}-11 \delta_{3}}+5 e^{-9 \delta_{1}-11 \delta_{3}}\right.$

$-3 e^{-8 \delta_{1}-11 \delta_{3}}+6 e^{-7 \delta_{1}-11 \delta_{2}}-6 e^{-6 \delta_{1}-11 \delta_{3}}-2 e^{-5 \delta_{1}-11 \delta_{3}}-2 e^{-4 \delta_{1}-11 \delta_{3}}-3 e^{-11 \delta_{1}-10 \delta_{3}}-5 e^{-9 \delta_{1}-10 \delta_{3}}+3 e^{-8 \delta_{1}-10 \delta_{3}}$

$+4 e^{-7 \delta_{1}-10 \delta_{3}}+10 e^{-6 \delta_{1}-10 \delta_{3}}-2 e^{-4 \delta_{1}-10 \delta_{3}}-4 e^{-3 \delta_{1}-10 \delta_{3}}+5 e^{-11 \delta_{1}-9 \delta_{3}}-5 e^{-10 \delta_{1}-9 \delta_{3}}-6 e^{-8 \delta_{1}-9 \delta_{3}}+5 e^{-7 \delta_{1}-9 \delta_{3}}$

$+3 e^{-6 \delta_{1}-9 \delta_{3}}+12 e^{-5 \delta_{1}-9 \delta_{3}}-16 e^{-4 \delta_{1}-9 \delta_{3}}-4 e^{-2 \delta_{1}-9 \delta_{3}}-3 e^{-11 \delta_{1}-8 \delta_{2}}+3 e^{-10 \delta_{1}-8 \delta_{3}}-6 e^{-9 \delta_{1}-8 \delta_{3}}+e^{-7 \delta_{1}-8 \delta_{3}}+3 e^{-6 \delta_{1}-8 \delta_{3}}$

$+12 e^{-4 \delta_{1}-8 \delta_{3}}-24 e^{-3 \delta_{1}-8 \delta_{3}}-4 e^{-\delta_{1}-8 \delta_{3}}+6 e^{-11 \delta_{1}-7 \delta_{3}}+4 e^{-10 \delta_{1}-7 \delta_{3}}+5 e^{-9 \delta_{1}-7 \delta_{3}}+e^{-8 \delta_{1}-7 \delta_{3}}+7 e^{-6 \delta_{1}-7 \delta_{3}}$

$+12 e^{-5 \delta_{1}-7 \delta_{3}}+8 e^{-4 \delta_{1}-7 \delta_{3}}+12 e^{-3 \delta_{1}-7 \delta_{3}}-16 e^{-2 \delta_{1}-7 \delta_{3}}-2 e^{-\delta_{1}-7 \delta_{3}}-6 e^{-11 \delta_{1}-6 \delta_{3}}+10 e^{-10 \delta_{1}-6 \delta_{2}}+3 e^{-9 \delta_{1}-6 \delta_{3}}$

$+3 e^{-8 \delta_{1}-6 \delta_{3}}+7 e^{-7 \delta_{1}-6 \delta_{3}}+4 e^{-5 \delta_{1}-6 \delta_{3}}+12 e^{-4 \delta_{1}-6 \delta_{3}}+12 e^{-2 \delta_{1}-6 \delta_{3}}-10 e^{-\delta_{1}-6 \delta_{3}}-2 e^{-11 \delta_{1}-5 \delta_{3}}+12 e^{-9 \delta_{1}-5 \delta_{3}}$

$+12 e^{-7 \delta_{1}-5 \delta_{3}}+4 e^{-6 \delta_{1}-5 \delta_{3}}+7 e^{-4 \delta_{1}-5 \delta_{3}}+3 e^{-3 \delta_{1}-5 \delta_{3}}+3 e^{-2 \delta_{1}-5 \delta_{3}}+10 e^{-\delta_{1}-5 \delta_{3}}-2 e^{-11 \delta_{1}-4 \delta_{3}}-2 e^{-10 \delta_{1}-4 \delta_{3}}$

$-16 e^{-9 \delta_{1}-4 \delta_{3}}+12 e^{-8 \delta_{1}-4 \delta_{2}}+8 e^{-7 \delta_{1}-4 \delta_{3}}+12 e^{-6 \delta_{1}-4 \delta_{3}}+7 e^{-5 \delta_{1}-4 \delta_{2}}+e^{-3 \delta_{1}-4 \delta_{3}}+5 e^{-2 \delta_{1}-4 \delta_{3}}+4 e^{-\delta_{1}-4 \delta_{3}}-4 e^{-10 \delta_{1}-3 \delta_{3}}$

$-24 e^{-8 \delta_{1}-3 \delta_{3}}+12 e^{-7 \delta_{1}-3 \delta_{3}}+3 e^{-5 \delta_{1}-3 \delta_{3}}+e^{-4 \delta_{1}-3 \delta_{3}}-6 e^{-2 \delta_{1}-3 \delta_{3}}+3 e^{-\delta_{1}-3 \delta_{2}}-4 e^{-9 \delta_{1}-2 \delta_{3}}-16 e^{-7 \delta_{1}-2 \delta_{3}}+12 e^{-6 \delta_{1}-2 \delta_{3}}$

$+3 e^{-5 \delta_{1}-2 \delta_{3}}+5 e^{-4 \delta_{1}-2 \delta_{3}}-6 e^{-3 \delta_{1}-2 \delta_{3}}-5 e^{-\delta_{1}-2 \delta_{3}}-10 e^{5\left(-\delta_{1}-2 \delta_{3}\right)}-4 e^{-8 \delta_{1}-\delta_{3}}-2 e^{-7 \delta_{1}-\delta_{3}}-10 e^{-6 \delta_{1}-\delta_{3}}+10 e^{-5 \delta_{1}-\delta_{3}}$

$+4 e^{-4 \delta_{1}-\delta_{3}}+3 e^{-3 \delta_{1}-\delta_{3}}-5 e^{-2 \delta_{1}-\delta_{3}}-10 e^{5\left(-2 \delta_{1}-\delta_{3}\right)}-5 e^{-\delta_{1}-\delta_{3}}-2 e^{2\left(-\delta_{1}-\delta_{3}\right)}-6 e^{3\left(-\delta_{1}-\delta_{3}\right)}-7 e^{4\left(-\delta_{1}-\delta_{3}\right)}-13 e^{5\left(-\delta_{1}-\delta_{3}\right)}$

$-13 e^{6\left(-\delta_{1}-\delta_{3}\right)}-7 e^{7\left(-\delta_{1}-\delta_{2}\right)}-6 e^{8\left(-\delta_{1}-\delta_{3}\right)}-2 e^{9\left(-\delta_{1}-\delta_{3}\right)}-5 e^{10\left(-\delta_{1}-\delta_{3}\right)}+e^{11\left(-\delta_{1}-\delta_{3}\right)}-2 e^{-7 \delta_{3}}-2 e^{-6 \delta_{3}}-6 e^{-5 \delta_{3}}+6 e^{-4 \delta_{3}}$

$\left.-3 e^{-3 \delta_{3}}+5 e^{-2 \delta_{3}}-3 e^{-\delta_{2}}\right)-1$,

$$
\begin{aligned}
F_{5}= & -\left(1+e^{-3 \delta_{1}}+e^{-2 \delta_{1}}+e^{-\delta_{1}}\right)\left(1+e^{-3 \delta_{3}}+e^{-2 \delta_{2}}+e^{-\delta_{3}}\right)\left(-4 e^{-9 \delta_{1}}+8 e^{-8 \delta_{1}}-8 e^{-7 \delta_{1}}+11 e^{-6 \delta_{1}}-16 e^{-5 \delta_{1}}+11 e^{-4 \delta_{1}}\right. \\
& -8 e^{-3 \delta_{1}}+8 e^{-2 \delta_{1}}-4 e^{-\delta_{1}}-4 e^{-9 \delta_{1}-10 \delta_{3}}+8 e^{-8 \delta_{1}-10 \delta_{3}}-8 e^{-7 \delta_{1}-10 \delta_{3}}+11 e^{-6 \delta_{1}-10 \delta_{3}}+11 e^{-4 \delta_{1}-10 \delta_{3}}-8 e^{-3 \delta_{1}-10 \delta_{3}} \\
& -4 e^{-\delta_{1}-10 \delta_{3}}+4 e^{-10 \delta_{1}-9 \delta_{3}}+4 e^{-6 \delta_{1}-9 \delta_{3}}+4 e^{-4 \delta_{1}-9 \delta_{3}}-8 e^{-10 \delta_{1}-8 \delta_{3}}-8 e^{-6 \delta_{1}-8 \delta_{3}}-8 e^{-4 \delta_{1}-8 \delta_{3}}+8 e^{-10 \delta_{1}-7 \delta_{3}}+8 e^{-6 \delta_{1}-7 \delta_{3}} \\
& +8 e^{-4 \delta_{1}-7 \delta_{3}}-11 e^{-10 \delta_{1}-6 \delta_{3}}-4 e^{-9 \delta_{1}-6 \delta_{3}}+8 e^{-8 \delta_{1}-6 \delta_{2}}-8 e^{-7 \delta_{1}-6 \delta_{3}}-16 e^{-5 \delta_{1}-6 \delta_{3}}-8 e^{-3 \delta_{1}-6 \delta_{3}}+8 e^{-2 \delta_{1}-6 \delta_{3}}-4 e^{-\delta_{1}-6 \delta_{3}} \\
& +16 e^{-6 \delta_{1}-5 \delta_{3}}+16 e^{-4 \delta_{1}-5 \delta_{3}}+8 e^{2\left(-\delta_{1}-5 \delta_{3}\right)}-11 e^{-10 \delta_{1}-4 \delta_{3}}-4 e^{-9 \delta_{1}-4 \delta_{3}}+8 e^{-8 \delta_{1}-4 \delta_{3}}-8 e^{-7 \delta_{1}-4 \delta_{3}}-16 e^{-5 \delta_{1}-4 \delta_{3}} \\
& -8 e^{-3 \delta_{1}-4 \delta_{3}}+8 e^{-2 \delta_{1}-4 \delta_{3}}-4 e^{-\delta_{1}-4 \delta_{3}}+8 e^{-10 \delta_{1}-3 \delta_{3}}+8 e^{-6 \delta_{1}-3 \delta_{3}}+8 e^{-4 \delta_{1}-3 \delta_{2}}-8 e^{-6 \delta_{1}-2 \delta_{3}}-8 e^{-4 \delta_{1}-2 \delta_{3}}-16 e^{5\left(-\delta_{1}-2 \delta_{2}\right)} \\
& +4 e^{-10 \delta_{1}-\delta_{3}}+4 e^{-6 \delta_{1}-\delta_{3}}-8 e^{2\left(-5 \delta_{1}-\delta_{2}\right)}+4 e^{-4 \delta_{1}-\delta_{3}}+16 e^{5\left(-2 \delta_{1}-\delta_{3}\right)}+4 e^{-9 \delta_{2}}-8 e^{-8 \delta_{3}}+8 e^{-7 \delta_{3}}-11 e^{-6 \delta_{3}}+16 e^{-5 \delta_{3}} \\
& \left.-11 e^{-4 \delta_{2}}+8 e^{-3 \delta_{3}}-8 e^{-2 \delta_{3}}+4 e^{-\delta_{3}}\right)
\end{aligned}
$$




$$
\begin{aligned}
& F_{6}=e^{-13 \delta_{1}}-3 e^{-12 \delta_{1}}+5 e^{-11 \delta_{1}}-3 e^{-10 \delta_{1}}+8 e^{-9 \delta_{1}}-4 e^{-8 \delta_{1}}+2 e^{-7 \delta_{1}}+2 e^{-6 \delta_{1}}+2 e^{-5 \delta_{1}}+2 e^{-4 \delta_{1}}+2 e^{-9 \delta_{1}-13 \delta_{3}}+2 e^{-8 \delta_{1}-13 \delta_{3}} \\
& +2 e^{-7 \delta_{1}-13 \delta_{3}}+2 e^{-6 \delta_{1}-13 \delta_{3}}-4 e^{-5 \delta_{1}-13 \delta_{3}}+8 e^{-4 \delta_{1}-13 \delta_{3}}-3 e^{-3 \delta_{1}-13 \delta_{2}}+5 e^{-2 \delta_{1}-13 \delta_{3}}-3 e^{-\delta_{1}-13 \delta_{3}}-6 e^{-9 \delta_{1}-12 \delta_{3}} \\
& -6 e^{-8 \delta_{1}-12 \delta_{3}}-10 e^{-7 \delta_{1}-12 \delta_{3}}-8 e^{-5 \delta_{1}-12 \delta_{3}}-7 e^{-\delta_{1}-12 \delta_{3}}+8 e^{-9 \delta_{1}-11 \delta_{3}}+8 e^{-8 \delta_{1}-11 \delta_{3}}+10 e^{-7 \delta_{1}-11 \delta_{3}}+10 e^{-6 \delta_{1}-11 \delta_{3}} \\
& -2 e^{-5 \delta_{1}-11 \delta_{3}}+10 e^{-4 \delta_{1}-11 \delta_{3}}+e^{-3 \delta_{1}-11 \delta_{3}}+9 e^{-2 \delta_{1}-11 \delta_{3}}+e^{-\delta_{1}-11 \delta_{3}}-4 e^{-9 \delta_{1}-10 \delta_{3}}-4 e^{-8 \delta_{1}-10 \delta_{3}}-6 e^{-7 \delta_{1}-10 \delta_{2}} \\
& -6 e^{-6 \delta_{1}-10 \delta_{3}}+6 e^{-4 \delta_{1}-10 \delta_{3}}-7 e^{-3 \delta_{1}-10 \delta_{3}}-7 e^{-\delta_{1}-10 \delta_{3}}+2 e^{-13 \delta_{1}-9 \delta_{3}}-6 e^{-12 \delta_{1}-9 \delta_{3}}+8 e^{-11 \delta_{1}-9 \delta_{3}}-4 e^{-10 \delta_{1}-9 \delta_{3}} \\
& +4 e^{-8 \delta_{1}-9 \delta_{3}}+3 e^{-7 \delta_{1}-9 \delta_{3}}+3 e^{-6 \delta_{1}-9 \delta_{3}}+3 e^{-5 \delta_{1}-9 \delta_{3}}-e^{-4 \delta_{1}-9 \delta_{2}}+6 e^{-3 \delta_{1}-9 \delta_{3}}+10 e^{-2 \delta_{1}-9 \delta_{3}}+4 e^{-\delta_{1}-9 \delta_{3}}+2 e^{-13 \delta_{1}-8 \delta_{3}} \\
& -6 e^{-12 \delta_{1}-8 \delta_{3}}+8 e^{-11 \delta_{1}-8 \delta_{3}}-4 e^{-10 \delta_{1}-8 \delta_{3}}+4 e^{-9 \delta_{1}-8 \delta_{3}}-e^{-7 \delta_{1}-8 \delta_{3}}-e^{-6 \delta_{1}-8 \delta_{3}}+7 e^{-5 \delta_{1}-8 \delta_{3}}+3 e^{-4 \delta_{1}-8 \delta_{3}}-6 e^{-3 \delta_{1}-8 \delta_{2}} \\
& -2 e^{-2 \delta_{1}-8 \delta_{3}}-8 e^{-\delta_{1}-8 \delta_{3}}+2 e^{-13 \delta_{1}-7 \delta_{3}}-10 e^{-12 \delta_{1}-7 \delta_{3}}+10 e^{-11 \delta_{1}-7 \delta_{3}}-6 e^{-10 \delta_{1}-7 \delta_{3}}+3 e^{-9 \delta_{1}-7 \delta_{3}}-e^{-8 \delta_{1}-7 \delta_{3}} \\
& -10 e^{-6 \delta_{1}-7 \delta_{3}}-e^{-5 \delta_{1}-7 \delta_{3}}+3 e^{-4 \delta_{1}-7 \delta_{3}}-6 e^{-3 \delta_{1}-7 \delta_{3}}+10 e^{-2 \delta_{1}-7 \delta_{2}}-10 e^{-\delta_{1}-7 \delta_{3}}+2 e^{-13 \delta_{1}-6 \delta_{3}}+10 e^{-11 \delta_{1}-6 \delta_{3}} \\
& -6 e^{-10 \delta_{1}-6 \delta_{3}}+3 e^{-9 \delta_{1}-6 \delta_{3}}-e^{-8 \delta_{1}-6 \delta_{3}}-10 e^{-7 \delta_{1}-6 \delta_{3}}-e^{-5 \delta_{1}-6 \delta_{3}}+3 e^{-4 \delta_{1}-6 \delta_{3}}-6 e^{-3 \delta_{1}-6 \delta_{3}}+10 e^{-2 \delta_{1}-6 \delta_{3}}-10 e^{-\delta_{1}-6 \delta_{3}} \\
& +e^{2\left(-\delta_{1}-6 \delta_{3}\right)}-4 e^{-13 \delta_{1}-5 \delta_{3}}-8 e^{-12 \delta_{1}-5 \delta_{3}}-2 e^{-11 \delta_{1}-5 \delta_{3}}+3 e^{-9 \delta_{1}-5 \delta_{3}}+7 e^{-8 \delta_{1}-5 \delta_{3}}-e^{-7 \delta_{1}-5 \delta_{2}}-e^{-6 \delta_{1}-5 \delta_{3}}+4 e^{-4 \delta_{1}-5 \delta_{3}} \\
& -4 e^{-3 \delta_{1}-5 \delta_{3}}+8 e^{-2 \delta_{1}-5 \delta_{3}}-6 e^{-\delta_{1}-5 \delta_{3}}+e^{2\left(-\delta_{1}-5 \delta_{3}\right)}+8 e^{-13 \delta_{1}-4 \delta_{3}}+10 e^{-11 \delta_{1}-4 \delta_{3}}+6 e^{-10 \delta_{1}-4 \delta_{3}}-e^{-9 \delta_{1}-4 \delta_{3}}+3 e^{-8 \delta_{1}-4 \delta_{3}} \\
& +3 e^{-7 \delta_{1}-4 \delta_{3}}+3 e^{-6 \delta_{1}-4 \delta_{3}}+4 e^{-5 \delta_{1}-4 \delta_{3}}-4 e^{-3 \delta_{1}-4 \delta_{3}}+8 e^{-2 \delta_{1}-4 \delta_{2}}-6 e^{-\delta_{1}-4 \delta_{3}}-7 e^{3\left(-\delta_{1}-4 \delta_{3}\right)}-3 e^{-13 \delta_{1}-3 \delta_{3}}+e^{-11 \delta_{1}-3 \delta_{3}} \\
& -7 e^{-10 \delta_{1}-3 \delta_{3}}+6 e^{-9 \delta_{1}-3 \delta_{2}}-6 e^{-8 \delta_{1}-3 \delta_{3}}-6 e^{-7 \delta_{1}-3 \delta_{3}}-6 e^{-6 \delta_{1}-3 \delta_{3}}-4 e^{-5 \delta_{1}-3 \delta_{3}}-4 e^{-4 \delta_{1}-3 \delta_{3}}+4 e^{4\left(-\delta_{1}-3 \delta_{2}\right)} \\
& +5 e^{-13 \delta_{1}-2 \delta_{3}}+9 e^{-11 \delta_{1}-2 \delta_{3}}+10 e^{-9 \delta_{1}-2 \delta_{2}}-2 e^{-8 \delta_{1}-2 \delta_{3}}+10 e^{-7 \delta_{1}-2 \delta_{3}}+10 e^{-6 \delta_{1}-2 \delta_{3}}+8 e^{-5 \delta_{1}-2 \delta_{3}}+8 e^{-4 \delta_{1}-2 \delta_{3}} \\
& -6 e^{5\left(-\delta_{1}-2 \delta_{2}\right)}-10 e^{6\left(-\delta_{1}-2 \delta_{3}\right)}-3 e^{-13 \delta_{1}-\delta_{3}}-7 e^{-12 \delta_{1}-\delta_{3}}+e^{-11 \delta_{1}-\delta_{3}}-7 e^{-10 \delta_{1}-\delta_{3}}+4 e^{-9 \delta_{1}-\delta_{3}}-8 e^{-8 \delta_{1}-\delta_{3}}-10 e^{-7 \delta_{1}-\delta_{3}} \\
& -10 e^{-6 \delta_{1}-\delta_{3}}+e^{2\left(-6 \delta_{1}-\delta_{3}\right)}-6 e^{-5 \delta_{1}-\delta_{3}}+e^{2\left(-5 \delta_{1}-\delta_{3}\right)}-6 e^{-4 \delta_{1}-\delta_{3}}-7 e^{3\left(-4 \delta_{1}-\delta_{3}\right)}+4 e^{4\left(-3 \delta_{1}-\delta_{3}\right)}-6 e^{5\left(-2 \delta_{1}-\delta_{3}\right)} \\
& -10 e^{6\left(-2 \delta_{1}-\delta_{2}\right)}+12 e^{4\left(-\delta_{1}-\delta_{3}\right)}-4 e^{5\left(-\delta_{1}-\delta_{3}\right)}-10 e^{6\left(-\delta_{1}-\delta_{3}\right)}-10 e^{7\left(-\delta_{1}-\delta_{3}\right)}-4 e^{8\left(-\delta_{1}-\delta_{3}\right)}+12 e^{9\left(-\delta_{1}-\delta_{3}\right)}+e^{-13 \delta_{3}}-3 e^{-12 \delta_{3}} \\
& +5 e^{-11 \delta_{3}}-3 e^{-10 \delta_{3}}+8 e^{-9 \delta_{3}}-4 e^{-8 \delta_{3}}+2 e^{-7 \delta_{3}}+2 e^{-6 \delta_{3}}+2 e^{-5 \delta_{3}}+2 e^{-4 \delta_{3}}, \\
& F_{7}=\left(e^{-3 \delta_{1}}+e^{-2 \delta_{1}}+e^{-\delta_{1}}+1\right)\left(-e^{4\left(-\delta_{1}-\delta_{2}\right)}-e^{6\left(-\delta_{1}-\delta_{3}\right)}+e^{10\left(-\delta_{1}-\delta_{3}\right)}+1\right)\left(e^{-3 \delta_{3}}+e^{-2 \delta_{3}}+e^{-\delta_{3}}+1\right)-1, \\
& F_{8}=\left(e^{-3 \delta_{1}}+e^{-2 \delta_{1}}+e^{-\delta_{1}}+1\right)\left(e^{-3 \delta_{3}}+e^{-2 \delta_{2}}+e^{-\delta_{3}}+1\right)\left[e^{4\left(-\delta_{1}-\delta_{3}\right)}\left(e^{-6 \delta_{3}}-e^{-6 \delta_{1}}\right)+e^{-6 \delta_{1}}+e^{-4 \delta_{1}}-e^{-6 \delta_{3}}-e^{-4 \delta_{3}}\right. \\
& \left.+e^{6\left(-\delta_{1}-\delta_{3}\right)}\left(e^{-4 \delta_{3}}-e^{-4 \delta_{1}}\right)\right] \\
& F_{9}=\left(e^{-3 \delta_{1}}+e^{-2 \delta_{1}}+e^{-\delta_{1}}+1\right)\left(e^{-6 \delta_{3}}-e^{-6 \delta_{1}}\right)\left(e^{-4 \delta_{3}}-e^{-4 \delta_{1}}\right)\left(e^{-3 \delta_{3}}+e^{-2 \delta_{2}}+e^{-\delta_{3}}+1\right) .
\end{aligned}
$$

${ }^{1}$ A. L. Velikovich, J. P. Dahlburg, A. J. Schmitt, J. H. Gardner, L. Phillips, F. L. Cochran, Y. K. Chong, G. Dimonte, and N. Metzler, Phys. Plasmas 7, 1662 (2000).

${ }^{2}$ C. Matsuoka and K. Nishihara, Phys. Rev. E 73, 055304(R) (2006).

${ }^{3}$ R. D. Richtmyer, Commun. Pure Appl. Math. 13, 297 (1960).

${ }^{4}$ E. E. Meshkov, Fluid Dyn. 4, 101 (1969).

${ }^{5}$ D. Arnett, Astrophys. J., Suppl. Ser. 127, 213 (2000).

${ }^{6} \mathrm{C}$. Wu, J. Geophys. Res. [Space Phys.] 105(A11), 7533, https://doi.org/ 10.1029/1999JA000197 (2000).

${ }^{7}$ B. A. Remington, J. Kane, R. P. Drake, S. G. Glendinning, K. Estabrook, R. London, J. Castor, R. J. Wallace, D. Arnett, E. Liang, R. McCray, A. Rubenchik, and B. Fryxell, Phys. Plasmas 4, 1994 (1997).

${ }^{8}$ S. W. Haan, Phys. Plasmas 2, 2480 (1995).

${ }^{9}$ Y. Yang, Q. Zhang, and D. H. Sharp, Phys. Fluids 6, 1856 (1994).

${ }^{10}$ A. N. Aleshin, E. V. Lazareva, S. G. Zaitsev, V. B. Rozanov, E. G. Gamalii, and I. G. Lebo, Sov. Phys. Dokl. 35, 159 (1990).

${ }^{11}$ R. Benjamin, D. Besnard, and J. Haas, LANL Report No. LA-UR 92$1185,1993$.

${ }^{12}$ G. Dimonte, Phys. Plasmas 6, 2009 (1999).

${ }^{13}$ D. Oron, L. Arazi, D. Kartoon, A. Rikanati, U. Alon, and D. Shvarts, Phys. Plasmas 8, 2883 (2001).
${ }^{14}$ J. W. Grove, R. Holmes, D. H. Sharp, Y. Yang, and Q. Zhang, Phys. Rev. Lett. 71, 3473 (1993).

${ }^{15}$ K. O. Mikaelian, Phys. Rev. Lett. 71, 2903 (1993).

${ }^{16}$ R. L. Holmes, J. W. Grove, and D. H. Sharp, J. Fluid Mech. 301, 51 (1995).

${ }^{17}$ U. Alon, J. Hecht, D. Ofer, and D. Shvarts, Phys. Rev. Lett. 74, 534 (1995).

${ }^{18}$ S.-I. Sohn, Phys. Rev. E 69, 036703 (2004).

${ }^{19}$ S. W. Haan, Phys. Fluids B 3, 2349 (1991).

${ }^{20}$ J. Hecht, U. Alon, and D. Shvarts, Phys. Fluids A 6, 4019 (1994).

${ }^{21}$ Q. Zhang and S.-I. Sohn, Phys. Lett. A 212, 149 (1996).

${ }^{22}$ S.-I. Sohn, Phys. Rev. E 67, 026301 (2003).

${ }^{23}$ J. G. Wouchuk, Phys. Rev. E 63, 056303 (2001).

${ }^{24}$ C. Mügler and S. Gauthier, Phys. Rev. E 58, 4548 (1998).

${ }^{25}$ A. Rikanati, U. Alon, and D. Shvarts, Phys. Rev. E 58, 7410 (1998).

${ }^{26}$ M. Vandenboomgaerde, S. Gauthier, and C. Mügler, Phys. Fluids 14, 1111 (2002).

${ }^{27}$ W. H. Liu, X. T. He, and C. P. Yu, Phys. Plasmas 19, 072108 (2012).

${ }^{28}$ C. M. Bender and S. A. Orszag, Advanced Mathematical Methods for Scientists and Engineers (McGraw-Hill, New York, 1978).

${ }^{29}$ K. O. Mikaelian, Phys. Rev. A 31, 410 (1985). 